\title{
Differential diagnosis and clinical management of diastolic heart failure: current best practice
}

This article was published in the following Dove Press journal:

Research Reports in Clinical Cardiology

29 September 2016

Number of times this article has been viewed

\author{
Justin Tawil' \\ Theresa A Gelzinis ${ }^{2}$ \\ 'Department of Anesthesiology, \\ Medical College of Wisconsin, \\ Milwaukee, WI, ${ }^{2}$ Department of \\ Anesthesiology, University of \\ Pittsburgh, Pittsburgh, PA, USA
}

Correspondence: Theresa A Gelzinis Department of Anesthesiology, University of Pittsburgh, 200 Lothrop Street, Pittsburgh, PA 15217, USA

$\mathrm{Tel}+\mathrm{I} 4 \mid 26473262$

Fax + I 4I26476290

Email gelzinista@anes.upmc.edu

\begin{abstract}
As the population ages, the prevalence of diastolic dysfunction and heart failure with preserved ejection fraction is rising. The presentation and management of these patients is increasing in frequency and requires an understanding of its pathophysiology, diagnostic methodology, as well as modern therapy for optimal outcome. This review will discuss the mechanisms, clinical stages, diagnostic grading, and therapeutic options for the management of diastolic dysfunction.
\end{abstract}

Keywords: heart failure, echocardiography, diastolic dysfunction, preserved ejection fraction

\section{Introduction}

With continued advances in population health and safety, life expectancy has steadily increased. Congestive heart failure is among those diseases growing in prevalence as the population ages. It is estimated that 870,000 new cases of heart failure will be diagnosed this year, approaching 10 per 1,000 population over 65 years of age. ${ }^{1}$ Diastolic dysfunction (DD) is defined as an increase in left ventricular (LV) stiffness with abnormal relaxation and filling. ${ }^{2,3}$ Hunt recently described DD as abnormal filling pressures in the setting of normal contractility. ${ }^{4}$ Though these abnormalities are present in patients with systolic heart failure, they can also be observed in patients with heart failure symptoms and a normal or near-normal ejection fraction (EF), described as diastolic heart failure or heart failure with preserved EF (HFPEF). This review will discuss the epidemiology of HFPEF, normal and abnormal cardiac relaxation, as well as the diagnosis and management of these patients.

HFPEF occurs when diastolic abnormalities impair the cardiac function, resulting in the inability to maintain the metabolic demands of the body, or requiring higher filling pressure to do so, producing symptoms of exercise intolerance and congestion. Systolic function, as measured by the LV ejection fraction (LVEF), is in the normal to near-normal $(>50 \%)$ range. Approximately half of the patients presenting with heart failure will be diagnosed with HFPEF, and as the population ages, this number is expected to rise.

While the clinical presentation, hospitalization rates, morbidity, and mortality of HFPEF are similar to those of heart failure with reduced EF (HFREF), there are many characteristics that are unique to HFPEF. HFPEF is more prevalent in women and more common with aging, obesity, and in African-Americans. Patients with HFPEF have more comorbidities than the patients with HFREF and are less likely to have obstructive 
coronary disease, but are more likely to have hypertension, obesity, and metabolic syndrome. ${ }^{5}$

\section{Epidemiology}

In the general population, the prevalence of DD ranges from $21.2 \%$ to $27.3 \%,{ }^{6,7}$ while the prevalence of HFPEF ranges from $1.1 \%$ to $1.5 \%{ }^{8}$ If left untreated, $\sim 12.2 \%$ of patients with DD will progress to overt heart failure. Risk factors for the progression to HFPEF include advanced age and an increased transmitral E to tissue Doppler e' $\left(\mathrm{E} / \mathrm{E}^{\prime}\right)$ ratio. ${ }^{6}$ Risk factors for the development of HFPEF include hypertension, advanced age, female sex, African-American race, and the presence of the metabolic syndrome. ${ }^{9}$ Other comorbidities associated in HFPEF include atrial fibrillation (AF), sleep apnea, chronic obstructive pulmonary disease, renal dysfunction, dyslipidemia, systemic inflammatory diseases, such as rheumatoid arthritis, and certain medications, including antineoplastic agents. ${ }^{10}$

\section{Etiology}

The two most common etiologies for HFPEF are hypertension and coronary artery disease (CAD). Other etiologies of HFPEF can be classified as systemic causes, such as obesity, diabetes mellitus, and advanced age; myocardial causes, including aortic stenosis, and cardiomyopathies such as hypertrophic obstructive; infiltrative diseases, including sarcoidosis; and pericardial causes, such as constrictive pericarditis and pericardial effusion ${ }^{11}$ (Table 1). Precipitating factors include ischemia, tachycardia, volume overload, arrhythmias,

Table I Etiologies of diastolic dysfunction

\begin{tabular}{l}
\hline Classification \\
\hline I. Systemic \\
Hypertension \\
Diabetes mellitus \\
Advanced age \\
Renal insufficiency \\
Thyrotoxicosis \\
II. Myocardial \\
Coronary artery disease \\
Valvular disease \\
Aortic stenosis \\
Cardiomyopathies \\
Hypertrophic obstructive \\
Restrictive \\
Idiopathic \\
III. Infiltrative \\
Hemochromatosis \\
Sarcoidosis \\
Idiopathic \\
IV. Pericardial \\
Pericardial effusion \\
Constrictive pericarditis
\end{tabular}

especially AF, and systemic stressors such as anemia and infection. ${ }^{12}$ Poor prognostic factors include advanced age, African-American race, and the presence of diabetes, renal disease, New York Heart Association (NYHA) functional class III or IV symptoms, and DD with restrictive filling. ${ }^{13}$

\section{Pathophysiology}

Diastole is defined as LV filling from the left atrium (LA) following the opening of a nonobstructed mitral valve and is affected by effective circulating plasma volume, the passive elastic properties of the ventricle, active relaxation, and the contribution from atrial contraction.

Diastole is an active process requiring adenosine triphosphate (ATP) and is mediated by the action of the cytoskeleton protein titin, an elastic protein that regulates passive tension, stiffness, and sarcomere stretch, and the intracellular regulation of calcium sensitivity and availability. Calcium sequestration into the sarcoplasmic reticulum is one of the major mechanisms of diastole. Normally, calcium is removed from the cytosol by three mechanisms: $70 \%$ of calcium removal occurs by the enzyme sarcoplasmic reticulum ATPase 2a (SERCA2a), 28\% through the sodium calcium exchanger (NCX), and the remainder by uptake into the mitochondria and removal through a plasma membrane $\mathrm{Ca}^{2+}$ ATPase. ${ }^{14}$

The activity of SERCA2a is regulated by the protein phospholamban (PLB). In its native state, PLB inhibits SERCA2a, while phosphorylation of PLB activates SERCA2a, causing calcium removal from the cytosol. ${ }^{15}$ The activity of PLB is regulated by calmodulin protein kinase II (CaMKII), the enzyme responsible for phosphorylating PLB.

In the sarcoplasmic reticulum (SR), the main calcium receptor is the ryanodine type 2-receptor (RyR2). The RyR2 is responsible for the massive release of calcium from the SR during the plateau phase of the action potential. Phosphorylation of this receptor by CaMKII increases the calcium sensitivity, causing the release of calcium and "hyperphosphorylation" of RyR2 by CaMKII. This makes the calcium "leak" out of the SR during diastole, which has been implicated in the development of AF and heart failure. ${ }^{16}$

Resting ventricular stiffness is mediated by the extracellular matrix and by the sarcomere spanning protein, titin. Titin, a cytoskeletal elastic protein that regulates passive tension, stiffness, and sarcomere stretch by functioning as a bidirectional spring, is responsible for early diastolic recoil and for creating the diastolic suction required for early filling and late diastolic resistance to stretch. ${ }^{17,18}$ Titin is present in two isoforms: the rigid $\mathrm{N} 2 \mathrm{~B}$ and the more compliant $\mathrm{N} 2 \mathrm{BA}$. The ratio and the phosphorylation state of these two isoforms determine the 
resting ventricular stiffness. Phosphorylation by protein kinase G (PKG) decreases N2B, while phosphorylation by CaMKII increases N2B, increasing the resting ventricular stiffness. ${ }^{19,20}$

The extracellular matrix also controls the ventricular stiffness by varying the type, amount, cross-linking, and degradation of collagen, and is mediated by degradation by matrix metalloproteinases (MMPs), including MMP-1, MMP-2, and MMP-9, and inhibition of collagen degradation by the tissue inhibitors of matrix metalloproteinases (TIMPs), especially TIMP-1. ${ }^{21}$

Nitric oxide (NO) and the natriuretic peptides (NPs) are also responsible for normal diastolic function. NO modulates $\beta$-adrenergic responsiveness and enhances LV relaxation and distensibility by reducing the myofilament sensitivity to calcium. ${ }^{22} \mathrm{NO}$ also activates guanylate cyclase, enhancing cyclic guanosine monophosphate generation and activating PKG. PKG enhances calcium reuptake into the SR, suppresses hypertrophic and fibrotic signaling, and stimulates LV relaxation and distensibility by phosphorylation of troponin I and stiff titin N2B. ${ }^{17}$ The NPs activate guanylate cyclase and stimulate SR cyclic guanosine monophosphate generation and $\mathrm{PKG}$ activation. ${ }^{23}$ Atrial and brain NPs stimulate natriuresis, vasodilation, and myocardial relaxation, while inhibiting neurohormonal activation, LV fibrosis, and hypertrophy. ${ }^{24,25}$

DD occurs due to increased LV stiffness and impaired LV relaxation.

\section{Increased LV stiffness}

Increased LV stiffness is the most common finding in HFPEF, and is due to changes in the isoform ratio and phosphorylation state of titin and also changes in the extracellular matrix. ${ }^{26-28}$ Increased $\mathrm{N} 2 \mathrm{~B}$ decreases the $\mathrm{N} 2 \mathrm{Ba} / \mathrm{N} 2 \mathrm{~B}$ ratio, resulting in impaired early diastolic filling and reduced compliance. ${ }^{19}$ Increased expression of the N2B isoform is seen with arterial stiffness, producing diastolic stiffness.

Increased myocyte growth is accompanied by increased connective tissue and myocardial stiffness. The degree of myocardial stiffness is determined by the composition and turnover rate of the extracellular matrix collagen accumulation, a change in the collagen phenotype, and by enhanced collagen cross-linking. ${ }^{29-31}$ This manifests as an increased fibrillar collagen content with altered geometry and an increased collagen I to III isotype ratio. ${ }^{32} \mathrm{LV}$ hypertrophy $(\mathrm{LVH})$, an adaptive response to pressure and volume overload, further exacerbates myocardial stiffness because the thickened myocardium is fibrotic. Instead of left ventricular hypertrophy $(\mathrm{LVH})$, patients with HFPEF may only have evidence of concentric remodeling, with an increase in wall thickness relative to cavity size. ${ }^{33-35}$ Prolonged LVH may contribute to cardiac dysfunction and a significant increase in the risk of cardiovascular disease. ${ }^{36}$ Severe $\mathrm{LVH}$ reduces subendocardial coronary flow reserve, further impairing diastolic function. ${ }^{12}$ In the Irbesartan in HFPEF (iPRESERVE) and Treatment of Preserved Cardiac Function Heart Failure with an Aldosterone Antagonist (TOP-CAT) trials, $>50 \%$ of patients either had LVH or concentric remodeling, and those patients tended to have poorer heart failure-related outcomes. ${ }^{37,38}$ Neurohormonal activation and the release of angiotensin II, aldosterone, and norepinephrine promote vasoconstriction, salt retention, cardiomyocyte hypertrophy, and fibrosis..$^{39,40}$ The inflammatory mediators interleukin (IL)-1 and IL-18 may contribute to LVH and fibrosis by the upregulation of osteopontin. ${ }^{41}$ Besides promoting fibrosis, IL-1 and IL-18 have negative lusitropic effects, further impairing diastolic function, suggesting that acute or chronic inflammation promotes DD. ${ }^{5}$

\section{Impaired LV relaxation}

LV relaxation is an active energy-requiring process, requiring ATP to release actin myosin crossbridges as well as for restoring the physiologic calcium concentration. Myocyte relaxation occurs when there is reduced myofibrillar sensitivity to calcium, occurring through the phosphorylation of troponin I by protein kinase A in an energy-dependent process. ${ }^{42}$ Patients with HFPEF have reduced myocardial energy reserve, thereby reducing the phosphorylation of troponin I, the rate of dissociation of calcium from troponin, and the dissociation of actin to myosin. Perturbations of ATP or calcium levels impair LV relaxation. ${ }^{14}$

During the plateau phase of the action potential, the extracellular calcium influx triggers a calcium-mediated calcium release by the ryanodine receptor (RyR2). RyR2 calcium efflux also occurs with $\beta$-stimulation by CaMKII. Phosphorylation of RyR2 at serine 2809 and 2815 increases RyR2 sensitivity to calcium, enhancing the transport during contraction and relaxation phases. ${ }^{43}$

The compressed form of titin, N2B, also facilitates the resolution of systole by desensitizing the actin-myosin complex to calcium. ${ }^{31}$ The stiff N2B is phosphorylated by protein kinase A, while the compliant N2BA is phosphorylated by CaMKII, which induces conformational changes in titin that reduce or increase the resting stiffness, respectively, as demonstrated in Figure $1 .{ }^{19,20}$ Ventricular stiffness is also affected by extracellular collagen cross-linking and degradation by metalloproteinases (MMP-1, -2, and -9), renin-angiotensin-aldosterone system (RAAS), and transforming growth factor beta (TGF- $\beta$ ). ${ }^{21}$ 


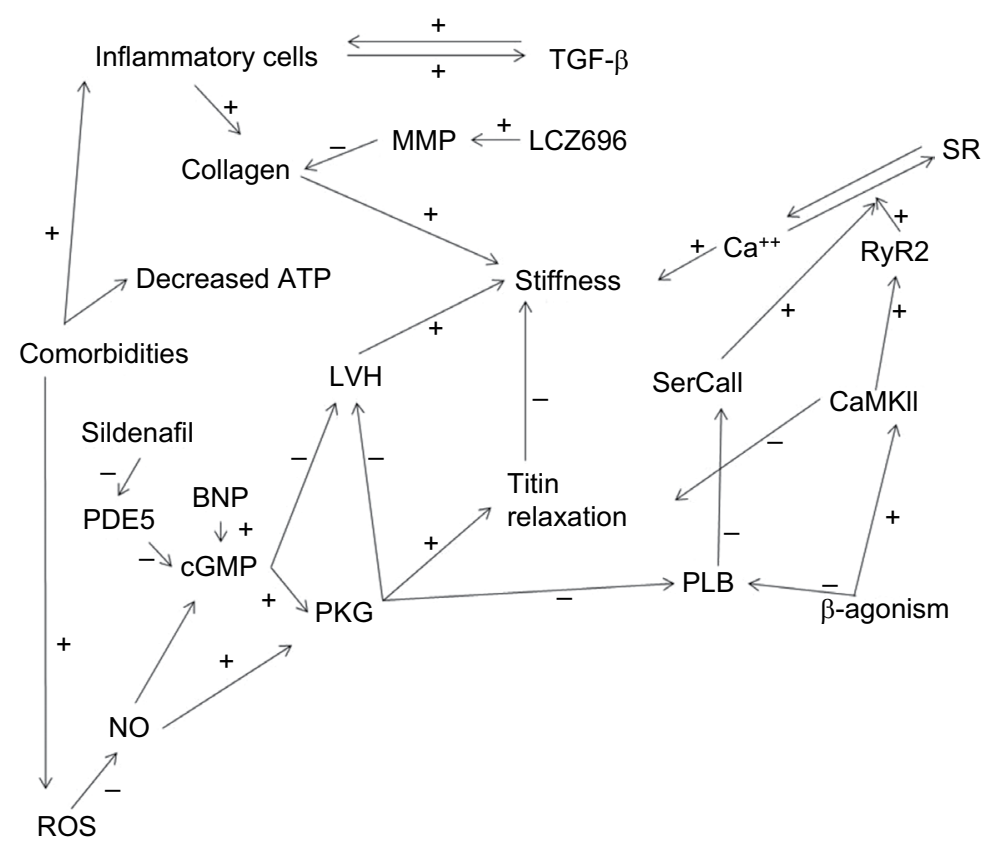

Figure I Major cellular mechanism of diastolic dysfunction.

Abbreviations: ATP, adenosine triphosphate; BNP, brain natriuretic peptide; cGMP, cyclic guanosine monophosphate; CaMKII, calmodulin protein kinase II; LVH, left ventricular hypertrophy; MMP, matrix metalloproteinase; NO, nitric oxide; PKG, protein kinase G; PLB, protein phospholamban; RyR2, ryanodine type 2-receptor; SR, sarcoplasmic reticulum; TGF- $\beta$, transforming growth factor beta; PDE5, Phosphodiesterase-5; ROS, reactive oxygen species.

Cardiac remodeling in HFPEF is different from that seen in systolic heart failure. Concentric ventricular hypertrophy and normal systolic function are the hallmarks of HFPEF. Comorbidities, especially obesity, increase the systemic inflammation, leading to endothelial dysfunction and an increase in oxygen-free radicals. ${ }^{28}$ These oxidative stress factors decrease $\mathrm{NO}$ and $\mathrm{PKG}$, while increasing the CaMKII activity, and in turn increasing the concentration of N2B and the resting ventricular stiffness. ${ }^{29}$ Increased muscle mass, interstitial fibrosis, hypertension, and aging reduce ventricular compliance and lead to reduced filling and higher resting pressures. RAAS activation leads to DD indirectly by increasing the afterload and inducing hypertrophic ventricular remodeling. Lower levels of PKG, which normally inhibit cellular hypertrophy, promote concentric hypertrophy. Relatively recent studies of the cellular structure and mechanism of DD in patients offer a unifying underlying mechanism behind the disparate comorbid conditions. ${ }^{34}$

Ventricular remodeling, endothelial dysfunction, reduction in NO, abnormal calcium handling, inflammation, and oxidative stresses all combine to reduce ventricular filling and compliance. ${ }^{44,45}$ Alterations in the extracellular matrix, increases in collagen deposition and cross-linking, as well as reduced elastin cause the aging, hypertensive, hypertrophied ventricles to stiffen. ${ }^{29,46,47}$ Collagen degradation and deposition is increased with elevated levels of circulating neurohormones and TGF- $\beta$. TGF- $\beta$ levels increase during myocardial inflammation and induce fibroblast differentiation to myofibroblasts. Myofibroblasts increase collagen deposition, inflammatory signal production, including IL-6 and IL-8, and monocyte chemoattractants. ${ }^{45}$ The downregulation of MMPs and upregulation of their inhibitors result in the aggregation of structural proteins and fibrosis. ${ }^{48,49}$ In addition to these myocyte structural changes, viral infections of coronary endothelial cells demonstrate their importance in normal diastolic function. ${ }^{50}$

In addition to structural changes, alterations in calcium handling increase ventricular stiffness. The decreased elimination of cytosolic calcium results in increased diastolic calcium and impaired relaxation. This increase in diastolic calcium is due to reduction in calcium transporter function, phosphorylation of PLB, and increased RyR2 calcium outflow. RyR2 receptors may open spontaneously, releasing calcium stores during diastole in patients with HFPEF. This efflux of calcium is exchanged for sodium through the NCX transporters, increasing the intracellular sodium and inducing uncoordinated spontaneous depolarization of resting cells. ${ }^{32}$ Even without frank depolarization, these elevations in cytosol sodium concentrations can facilitate diastolic calcium influx through NCX channels. ${ }^{51,52}$ This increase in cytosolic calcium during diastole reduces the available intracellular calcium required for contraction during systole, resulting in subclinical systolic dysfunction. 
Insulin resistance and obesity alter cellular glucose availability and reduce ATP availability during ischemia. ${ }^{53}$ This reduction in ATP further impairs the calcium transport channels due to limited fatty acid metabolism. Taken together, these alterations in hormonal, structural, ion transport, energy use, and coordination all lead to increased structural and functional ventricular stiffness. ${ }^{54}$ In addition, hyperglycemia damages the mitochondria, leading to additional free-radical burden. ${ }^{55}$ Similar pathways are activated in chronic hypertension. ${ }^{49}$

The pathophysiology of HFPEF involves increased LV and arterial stiffness, metabolic energy derangements, and ventricular systolic and diastolic abnormalities, resulting in abnormal LV relaxation and increased LV end-diastolic and pulmonary artery pressures. These disturbances first appear after exercise or are exacerbated by exercise, and are compounded by impaired chronotropic responses, systemic vasodilation, and heart rate recovery following exercise, as well as impaired atrial functional reserve. The resultant increase in LA pressure is transmitted to the pulmonary veins, producing pulmonary arteriole vasoconstriction, vascular remodeling, and fixed postcapillary pulmonary hypertension. Postcapillary pulmonary hypertension is common in HFPEF. ${ }^{56}$ The resultant increase in right ventricular afterload impairs the forward flow, further impairing LV filling.

Abnormal diastolic filling can be found in patients before the development of LVH or a change in systolic function. Mechanisms contributing to abnormal diastolic filling include stiffening of large arteries, hypertension (HTN), ischemia, diabetes, and myocardial fibrosis. Vascular endothelial dysfunction contributes to the development of DD through impaired NO production, increased NO degradation, and hyporesponsiveness in vascular smooth muscle. ${ }^{57,58}$

Patients with HFPEF have abnormal LV relaxation and increased LV stiffness, shifting the diastolic pressure-volume relationship upward and to the left (Figure 2). During exercise, there is increased LV stiffness, producing increased end-diastolic pressure and decreased stroke volume..$^{59}$ Other abnormalities during exercise include reduction in both systolic and diastolic function, increased pulmonary capillary wedge pressure with a low stroke volume index and work rate, and an increase in end-diastolic pressure (EDP) and elastance due to impaired relaxation, leading to exercise intolerance. ${ }^{60}$ This increase in elastance can reduce the LVEF, stroke volume, and cardiac output. ${ }^{61}$ Besides impaired passive relaxation, there is also reduced active relaxation, decreasing the LV filling during exercise, and failure of the Frank-Starling mechanism. ${ }^{62}$ Patients with low LV diastolic function reserve

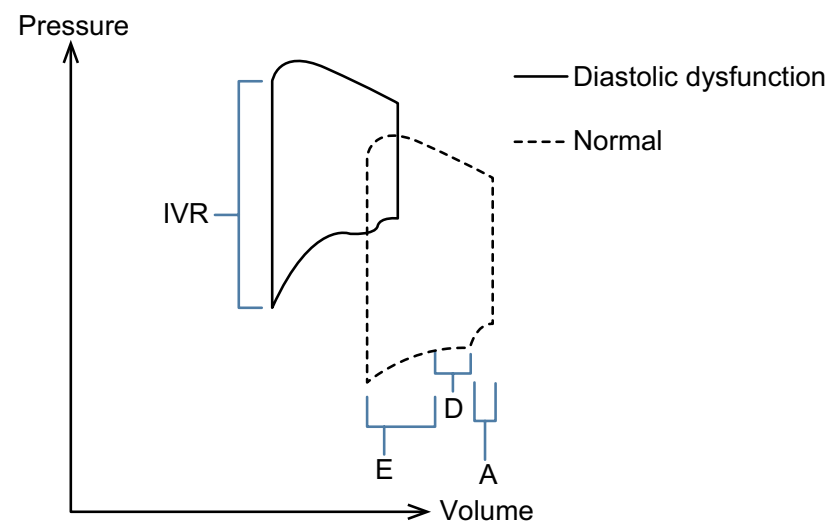

Figure 2 Phases of diastole.

Notes: The solid line represents diastolic dysfunction with a steeper pressurevolume relationship shifted up and to the left. The dotted line represents normal. E, $D$, and $A$ are labeled as is IVR.

Abbreviations: A, atrial contraction; D, diastasis; E, early filling; IVR, isovolumetric relaxation.

index had higher LV diastolic elastance during exercise and reduced exercise capacity. ${ }^{63}$

To compensate for the reduced filling due to slowed relaxation during early diastole, the LV is more dependent on atrial contraction. The LA can partially compensate the decrease in early diastolic filling until atrial dilation and failure occurs in the later stages of the disease.

Another result of the increased stiffness is ventricularvascular uncoupling. Ventricular-vascular coupling (VVC) is the interaction between the heart and the vasculature, and is essential to achieve maximal cardiac work, power, and chamber efficiency while maintaining the blood pressure and the cardiac output. ${ }^{64} \mathrm{VVC}$ is defined as the ratio of arterial to end-systolic elastance, as calculated from the slope of the end-systolic pressure-volume relation and the ratio of end-systolic pressure/stroke volume. In normal patients, exercise is usually associated with a reduction in systemic vascular resistance. In patients with HFPEF, the resting VCC is lower during exercise, resulting in an inappropriate increase in systemic vascular resistance, impairing LV emptying during systole precipitated by the increase in afterload. ${ }^{5,65}$

Other clinical manifestations include impaired chronotropic and systemic vasodilator responses to exercise, as well as reduced cardiac output reserve and blunted baroreceptor sensitivity. ${ }^{66}$ These patients also exhibit volume overload with anemia, renal dysfunction, and obesity. ${ }^{67}$

Patients with HFPEF have increased plasma volume due to neurohormonal activation of the RAAS and arginine vasopressin systems. This can manifest as acute decompensated cardiac failure either due to gradual fluid accumulation and worsening symptoms or, more commonly, as acute hypertensive failure characterized by hypertension, decreased 
contractility, afterload mismatch, and diastolic LV failure. ${ }^{64}$ The combination of LV and arterial stiffness, shifting the diastolic pressure-volume relationship upward and to the left, increases the sensitivity to both preload and afterload. A small increase in volume significantly increasing the LVEDP and/or an increase in afterload produces afterload mismatch, slowing LV active relaxation and transmitting these elevated pressures to the pulmonary veins and lungs, precipitating pulmonary edema. ${ }^{68}$

While the resting LVEF may appear normal in patients with HFPEF, during exercise or with increased venous return, the LVEF may fail to increase or even decrease, reflecting impaired contractile reserve or inducible systolic dysfunction. The inability of the LVEF and cardiac output to increase during exercise is a key feature of heart failure, known as impaired contractile reserve. ${ }^{69,70}$ Mechanisms for impaired contractile reserve include the presence of a small LV cavity, decreasing both stroke volume and end-systolic volume, the inability to increase end-systolic volume during exercise, ${ }^{71}$ $\beta$-receptor desensitization, either due to overstimulation by neurohormonal activation or downregulation of $\beta$-receptors ${ }^{72}$ or systemic inflammation, causing cytokine-mediated desensitization of $\beta$-receptors. ${ }^{73}$ These mechanisms contribute to exercise intolerance and produce a blunted response to physical exertion and $\beta$-agonists. ${ }^{74}$

HFPEF is also associated with reduced aerobic capacity, as measured by peak oxygen consumption $\left(\mathrm{VO}_{2}\right)$. A reduced peak $\mathrm{VO}_{2}$ is a result of a blunted exercise-induced increase in cardiac output and a diminished ability to extract and utilize oxygen for energy production in skeletal muscle. ${ }^{75}$ Ventilator inefficiency contributes to the heart failure symptoms and diminished aerobic capacity. There is an abnormal mismatch between minute ventilation and carbon dioxide production, leading to an elevated slope of the $\mathrm{VE} / \mathrm{VCO}_{2}$ curve. ${ }^{76}$ An abnormal $\mathrm{VE} / \mathrm{VCO}_{2}$ slope is also associated with postcapillary pulmonary hypertension in all types of heart failure. An end diastolic volume (EDV) $<97 \mathrm{~mL} / \mathrm{m}^{2}$ is required to establish a diagnosis of HFPEF. ${ }^{8,77}$ Primary HFPEF cannot be diagnosed in the presence of severe mitral and aortic valvular disease, LV outflow traction obstruction, as seen in hypertrophic or hypertensive cardiomyopathy, and impaired LV filling due to tachyarrhythmias.

\section{Diagnosis}

HFPEF is a heterogeneous syndrome that encompasses cardiovascular, metabolic, and proinflammatory diseases associated with advanced age and extracardiac comorbidities and is a diagnosis of exclusion. Patients with HFPEF are usually older, African-Americans, females, and present with obesity, diabetes, $\mathrm{CAD}$, and hypertension, the most common comorbidities associated with HFPEF. ${ }^{78,79}$ The presence of LVH is a component of the European Society of Cardiology guidelines for the diagnostic pathway for HFPEF. ${ }^{80}$ Atrial fibrillation occurs in approximately one-third of patients with HFPEF. It is more prevalent in decompensated heart failure regardless of the type; but compared to HFREF, AF is more frequent in patients with HFPEF. ${ }^{81,82}$ The high filling pressures associated with a stiff noncompliant ventricle lead to LA enlargement, resulting in AF. The end result is a loss of atrial contraction, decreased late diastolic filling, and a reduced cardiac output. ${ }^{83}$ $\mathrm{AF}$ is also associated with increased LV fibrosis, which can contribute to the development of DD. ${ }^{84} \mathrm{AF}$ was associated with adverse cardiovascular outcomes in the Candesartan in Heart failure - Assessment of Reduction in Mortality and morbidity (CHARM) study ${ }^{85}$ Ischemic heart disease is seen in HFPEF, but is more prevalent in HFREF. Ischemia contributes to HFPEF by increasing the LVEDP and myocardial stiffness. Diabetes mellitus (DM) is reported to be between $11 \%$ and $70 \%{ }^{86}$ In the Framingham Health Study, diabetes was associated with a steeper increase in LV mass and wall thickness. In the CHARM study, a diagnosis of DM was associated with a poorer outcome regardless of the EF and there was a greater relative risk of developing HFPEF than HFREF in diabetics. ${ }^{87}$ There is also a higher incidence of obesity in HFPEF patients than in patients with HFREF. Obesity can cause several symptoms found in HFPEF, including fatigue, dyspnea, and ankle swelling, and can cause obstructive sleep apnea (OSA), resulting in pulmonary hypertension and cor pulmonale. OSA can also account for several comorbidities seen in HFPEF, including HTN, LVH, diastolic impairment, and $\mathrm{AF}^{88}$ Obese patients without OSA have been shown to have impaired diastolic function, when compared to normal patients. ${ }^{89}$ Pulmonary hypertension is associated with worse survival in HFPEF. The Prevention of Renal and Vascular Endstage Disease (PREVEND) study recruited 8,592 participants to determine the risk of developing heart failure (HF) in this patient population. ${ }^{90}$ Three hundred and seventy-four cases of HF were described and of these, 125 were HFPEF. Female sex, older age, AF, increased urinary albumin excretion, and cystatin $\mathrm{C}$ were associated with a greater risk for developing HFPEF. In the Framingham Heart Study, 56,340 patients were followed over a 27 -year period; it was found that AF, a higher body mass index, and smoking were associated with an increased risk of HFPEF. ${ }^{91}$ A subset of this study also determined that airflow obstruction and asymptomatic baseline LV DD were associated with the development of HFPEF. ${ }^{92}$ Patients 
with HFPEF are more likely to die from noncardiovascular causes than the patients with HFREF, most likely due to the presence of multiple comorbidities that are associated with higher rates of noncardiovascular hospitalization and death. This may be why all the pharmacologic randomized controlled trials in patients with HFPEF have failed to show an improvement in overall mortality or hospitalization for heart failure. ${ }^{93}$

\section{Differential diagnosis}

Patients with HFPEF typically present either in acute heart failure with signs of pulmonary or peripheral congestion or with symptoms of breathlessness, fatigue, or exercise intolerance. In many cases, the signs and symptoms of HFPEF and HFREF are indistinguishable. Patients who present with HFPEF are usually older, females, obese, or AfricanAmericans, with diabetes mellitus and systolic hypertension, and are less likely to present with ischemia. Another sign more commonly seen with HFPEF is AF. Patients with abnormalities in diastolic function rely on atrial contraction for filling, and the development of AF, resulting from LA dilation, decreases the ventricular filling and cardiac output, producing pulmonary edema.

The differential diagnosis of HFPEF depends on the presentation. The differential in patients presenting with acute heart failure in the emergency department includes cardiac or respiratory etiologies, extracardiac fluid overload, or a high-output state. In patients presenting as outpatients with breathlessness or exercise intolerance, obesity and physical deconditioning can be included in the differential. ${ }^{86}$

Cardiac conditions in the differential of HFPEF include HFREF with an overestimation of the EF, valvular disease, atrial myxoma, pericardial disease, restrictive or hypertrophic cardiomyopathy, intracardiac shunt, severe hypertension, and acute coronary syndrome complicated by pulmonary edema. Echocardiography can be used to differentiate HFPEF from these conditions; but in patients with poor acoustic windows, other imaging modalities, such as cardiac magnetic resonance imaging (CMR), catheterization, or myocardial biopsy, may be required. Other indicators of HFPEF in the outpatient setting include ischemia without anginal pain, chronotropic incompetence, and dynamic mitral regurgitation; in these cases, exercise testing or stress echocardiography may be useful in diagnosing HFPEF.

Respiratory diseases may be difficult to distinguish from HFPEF because of the overlap between pulmonary hypertension, chronic obstructive pulmonary disease (COPD), and HFPEF. ${ }^{56} \mathrm{COPD}$ and other chronic lung diseases are prevalent in patients with $\mathrm{HFPEF}$, and pulmonary hypertension can be a component of both. COPD can cause cor pulmonary with right ventricular failure, presenting as heart failure with the levels of NPs not diagnostic for a heart failure diagnosis. ${ }^{94}$ Studies that may be useful to distinguish respiratory diseases from HFPEF include lung function tests, ventilation perfusion scanning, high-resolution computed tomography, sleep studies, and right heart catheterization.

Other diagnoses in the differential include conditions that produce extracardiac volume overload, such as nephrotic syndrome, anemia, hypothyroidism, arteriovenous fistula, or extracardiac shunting. If shortness of breath and fatigue are the initial presentation, obesity and physical deconditioning should be considered. In patients presenting initially with shortness of breath, HFPEF can be diagnosed using brain natriuretic peptide (BNP) and echocardiography. An NTproBNT of $>120 \mathrm{pg} / \mathrm{mL}$ or BNP of $>100 \mathrm{pg} / \mathrm{mL}$ without evidence of pulmonary disease should prompt further study with echocardiography to rule out valvular and pericardial disease, as well as HFREF, which would be diagnosed with an $\mathrm{EF}<50 \%$. Other echocardiographic findings suggestive of HEPEF include an left ventricular end-diastolic index $<76 \mathrm{~mL} / \mathrm{min}^{2}$, a LA volume indexed to body surface area $<29 \mathrm{~mL} / \mathrm{m}^{2}$ without $\mathrm{AF}$, and a left ventricular mass index $<96 \mathrm{~g} / \mathrm{m}^{2}$ in women and $<113 \mathrm{~g} / \mathrm{m}^{2}$ in men. A left ventricular end-diastolic volume index $>76 \mathrm{~mL} / \mathrm{min}$ is suggestive of a high-output state. Tissue Doppler can be used to distinguish between HFPEF and CAD with deficient angina warning. In HFPEF, the tissue Doppler imaging (TDI) $\mathrm{S}$ wave is $<6.5 \mathrm{~cm} / \mathrm{s}$ and the $\mathrm{E} / \mathrm{E}^{\prime}$ is $>8 .^{89}$

Since the first symptom of HFPEF is often exercise intolerance, patients presenting as outpatients may be asymptomatic with normal echocardiographic values at rest. Stress echocardiography can be useful in revealing exercise-induced diastolic impairment, dynamic mitral regurgitation, or silent ischemia. ${ }^{95}$ Other causes of heart failure, especially HFREF, should be sought out to provide optimal treatment.

The problem with diagnosing DD and HFPEF is that there is no standard definition to describe these disease entities. Selmeryd et al examined how the classifications of DD by the European Association of Cardiovascular Imaging and the American Society of Echocardiography are used and how these variations affect the reported prevalence of DD in the community. ${ }^{96}$ The problem with the European Association of Cardiovascular Imaging/American Society of Echocardiography algorithms is that there are no guidelines to deal with discordant measurements, leading to confusion when cardiologists were given the same premeasured variables. ${ }^{97,98}$ There were also large differences in HFPEF 
definitions and methods of using the algorithm between studies. ${ }^{99,100}$ Selmeryd et al performed a systematic review of studies utilizing diastolic dysfunction guidelines as set by the European Association of Cardiovascular Imaging/ American Society of Echocardiography (EACVI/ASE) and found discrepancies in the classification tree (one-level vs two-level) used. They also found that others did not grade the severity of DD or HFPEF. ${ }^{96}$ The other problem with the algorithm is that there is no guidance on how the parameters in the algorithm should be weighed.

According to the European Society of Cardiology criteria, the diagnosis of HFPEF is based on the presence of heart failure symptoms in association with hemodynamic, Doppler echocardiographic, or biomarker evidence of abnormal LV relaxation, filling, distensibility, or stiffness. The clinical spectrum of this disease begins with DD and progresses to HFPEF.

\section{Diastolic dysfunction}

DD refers to abnormalities of active relaxation and passive ventricular filling. Typically, these abnormalities are attributed to delayed or incomplete myocardial relaxation or tissue elasticity, but may also result from pericardial constriction. There are four recognized grades of DD that can be diagnosed using two-dimensional (2D), pulsed wave Doppler and TDI. 2D echocardiography is used to assess LA size and volume, EF, and presence of LVH. Examination of the transmitral inflow pattern using pulsed wave Doppler measures the early (E) and late (A) flow velocities, the deceleration time (DT), the time from the peak $\mathrm{E}$ wave to the baseline, and the isovolumic relaxation time (IVRT), the time between aortic valve closure and mitral valve opening. TDI is used to measure the motion of the mitral valve annulus. The grades of DD and the presence of elevated filling pressures can further be determined utilizing pulmonary vein flow, provocation testing, propagation velocity, and speckle tracking of diastolic strain rate.

LA volume is a measure of DD and can be used to assess the risk of myocardial infarction, stroke, $\mathrm{AF}$, and heart failure. ${ }^{101} \mathrm{~A}$ left atrial volume of $28-33 \mathrm{~mL} / \mathrm{m}^{2}$ is graded as mild, $34-39 \mathrm{~mL} / \mathrm{m}^{2}$ as moderate, and $>40 \mathrm{~mL} / \mathrm{m}^{2}$ as severe. ${ }^{102}$ The transmitral inflow velocities characterize LV filling and are used to differentiate the grades of DD. The early mitral inflow velocity, or E, is influenced by the LA/LV pressure gradient and is dependent on LA pressure, LV compliance, and the rate of LV relaxation. ${ }^{101}$ The A velocity is produced by atrial contraction and is influenced by LV compliance and LA contractility. The DT of the E wave correlates with the time it takes to equalize pressures between the LA and LV. Reduced equilibration times will shorten the DT, while longer times will increase it. Impaired relaxation lengthens the IVRT, while reduced compliance and increased LV filling pressures shorten it. The IVRT is affected by heart rate and ventricular function and although it is not useful as a single test to detect abnormalities in LV filling pressures, it may be useful in following the response to treatment.

Doppler values for normal diastolic function include an E/a ratio of 0.9-1.5, a DT of 160-240 ms, and an IVRT of $70-90 \mathrm{~ms}$. The first stage of DD is grade I or mild and is characterized by impaired relaxation and a reduction in the velocity of mitral annular relaxation. Normally, there are regional differences in myocardial relaxation. Relaxation starts in the apex and moves toward the base, and this apex-to-base pressure gradient is responsible for the diastolic suction required for early diastolic filling. ${ }^{46}$ This slowed relaxation causes the ventricular chamber pressures to decrease at a reduced rate, prolonging the time to minimum diastolic pressure compared with normal patients, reducing the LA/LV pressure gradient, and resulting in decreased passive, or early, diastolic filling. At this stage, late ventricular filling by atrial contraction is only minimally affected, producing late filling velocities that supersede early filling. Stage I DD manifests as a reversal of the E/A ratio $<0.9$ and a DT $>240 \mathrm{~ms}$. As long as there is adequate LV filling, intact atrial contraction, and an adequate diastolic filling period, LV pressures remain normal. The only clinical manifestation is reduced diastolic reserve and an inability to increase the rate of LV relaxation during exercise. There is a subset of patients with an A wave dominant transmitral inflow velocity and elevated filling pressures, as measured by an increased LA volume and an increased septal $\mathrm{E} / \mathrm{E}^{\prime}$ ratio of $>15$, which is known as stage Ia.

The next stage is grade II or moderate DD, also referred to as pseudonormal. As DD progresses, the LA pressure rises to a point where the pressure gradient between the LA and LV is restored, allowing early filling despite slowed LV relaxation. Despite the increase in early diastolic filling, LV relaxation peaks late during diastole. This late peak may result in the appearance of a characteristic L-wave prior to atrial contraction. ${ }^{103}$ The pressures that impede forward flow are reflected back to the LA and pulmonary veins. This returns the transmitral flow pattern to an E-wave dominant pattern and decreases the DT. This phenomenon is described as pseudonormalization, and can be differentiated from a normal pattern by the $\mathrm{e}^{\prime}$ and $\mathrm{E} / \mathrm{E}^{\prime}$, as measured with TDI, the LA volume, provocative testing with Valsalva or a nitroglycerin challenge, propagation velocity, or speckled tracking.

The mitral annular motion or $\mathrm{E}^{\prime}$, as measured by TDI, is useful in distinguishing between type II DD and a normal transmitral pattern. Normally, mitral inflow is initiated with rapid LV relaxation and suction of blood into the LV. When 
this occurs, the onset of $\mathrm{E}^{\prime}$ will either be slightly before or simultaneous with the onset of the transmitral E. Reduced early LV relaxation decreases the mitral annular motion or $\mathrm{E}^{\prime}$. A septal $\mathrm{E}^{\prime}<8 \mathrm{~cm} / \mathrm{s}$ or a lateral $\mathrm{e}^{\prime}<10 \mathrm{~cm} / \mathrm{s}$ is associated with reduced $\mathrm{LV}$ relaxation. If LA pressure is elevated and $L V$ relaxation reduced, the $E$ velocity may precede the onset of $E^{\prime} \cdot{ }^{104}$ A limitation of $E^{\prime}$ is that it is obtained from a single site on the mitral valve annulus and the assumption is that the $\mathrm{E}^{\prime}$ reflects global relaxation. Pulmonary vein flow is of lesser value in the diagnosis of DD. In patients with moderate-to-severe DD, there is a blunting of the $\mathrm{S}$ wave, indicative of elevated left atrial pressure (LAP). In patients with a low $\mathrm{E} / \mathrm{E}^{\prime}$ ratio, pulmonary vein flow duration greater than mitral inflow duration at atrial contraction may indicate an earlier stage of reduced LV compliance and increased LV end-diastolic pressure. The mitral inflow propagation velocity $(\mathrm{Vp})$ indirectly measures the degree of diastolic suction by measuring the velocity of the mitral valve inflow jet. The propagation velocity is obtained using color M-mode with the cursor in the direction of the mitral inflow jet. ${ }^{105-107}$ The $\mathrm{Vp}$ is measured as the slope of the line of the first aliasing velocity from the mitral valve to $4 \mathrm{~cm}$ distal to the $L V$, with a normal $\mathrm{Vp}$ of $>50 \mathrm{~cm} / \mathrm{s}$. The $\mathrm{Vp}$ is a measure of ventricular relaxation, but is inaccurate in hypertrophic and dilated LVs. 2D speckle tracking can be used to detect global myocardial motion, with less interference by annular and valvular pathology. The strain rate during isovolumic relaxation (SRivr) is a measure of global myocardial relaxation and may be a promising new tool in the evaluation of DD. Stage II $\mathrm{DD}$ is associated with decreased $\mathrm{E}^{\prime}$, increased $\mathrm{E} / \mathrm{E}^{\prime}$ ratio, and an $\mathrm{LV}$ volume $>28 \mathrm{~mL} / \mathrm{m}^{2}{ }^{108,109}$

Besides assessing for DD, the $\mathrm{E} / \mathrm{E}^{\prime}, \mathrm{E} / \mathrm{Vp}$, and $\mathrm{E} / \mathrm{SVivr}$ ratios can be used to detect elevated mean pulmonary capillary wedge pressures (PCWP). An E/E' $>15$ indicates elevated mean PCWP, while an $\mathrm{E} / \mathrm{E}^{\prime}<8$ indicates a normal PWCP. The mitral E wave/SRivr ratio is more sensitive in detecting increases in the mean PCWP, when compared to the $\mathrm{E} / \mathrm{E}^{\prime}$ ratio, especially in the patients with an $E / E^{\prime}$ of $8-15 .{ }^{110} \mathrm{An}$ E/SVivr ratio of $>236$ is indicative of elevated mean PCWP. The ratio of $\mathrm{E} / \mathrm{Vp}$ can also be used to estimate $\mathrm{LV}$ filling pressure, with an E/Vp $>1.5$ suggestive of a PCWP $>15 .{ }^{111,112}$

The next grade of DD is grade IIIa, also known as severe or reversible restrictive. At this stage, the atrial pressures have risen even further, such that early filling can resume despite the higher early diastolic LV pressure. The late diastolic pressures have become so elevated that even a coordinated atrial contraction generates only a relatively small pressure gradient. Atrial contraction against the higher LV end-diastolic pressures results in only modest forward flow, but does demonstrate an increase in retrograde pulmonary vein flow. ${ }^{113}$ As a result, the transmitral flow pattern demonstrates accelerated E-velocities, resulting in an E/A ratio of $>2 \mathrm{~cm} / \mathrm{s}$ and a DT $<160 \mathrm{~ms}$. The high-pressure chambers demonstrate higher E-velocities and equilibration is rapid between the stiff chambers. In these patients, provocation with nitroglycerin or a Valsalva maneuver reverts from the restrictive pattern to a grade I mitral valve inflow pattern. Clinically, these patients are likely to present with symptomatic heart failure at rest. The last and most severe grade of DD is grade IIIb, otherwise known as grade IV or fixed restrictive DD. The primary feature differentiating this stage from grade IIIa is the response to provocative testing. In all previous stages of dysfunction, transmitral flow patterns can be downgraded during conditions of decreased loading. Valsalva maneuvers and nitroglycerin administration temporarily reduce central venous return and, thus, atrial pressures. These temporary changes are reflected in the transmitral flow patterns as a reversion to an $\mathrm{A}>\mathrm{E}$ waveform. In grade $\mathrm{IIIb}$ disease, the restrictive pattern does not change during provocation and is associated with a poor prognosis.

Figure 3 describes the echo findings associated with the different stages of DD.

\section{Clinical evaluation}

The diagnosis of HFPEF begins with the history and physical exam to determine the presence of HF and to assess for concomitant disease. The history and physical exam itself cannot differentiate between HFPEF and HFREF. With regards to symptomatology, comorbid conditions in patients with HFPEF may contribute to the symptom burden by further impairing exercise tolerance, by reducing the threshold for symptoms, or by producing signs or symptoms that may be confused with HFREF. The patient's quality of life is affected not only by limitations in the activities of daily living but also by documented cognitive dysfunction. ${ }^{114}$

The symptoms of heart failure include shortness of breath, orthopnea or paroxysmal nocturnal dyspnea, fatigue, and reduced exercise tolerance. Clinical findings include elevated jugular venous pressure, hepatojugular reflux, third heart sound, laterally displaced apical impulse, a murmur, and peripheral or pulmonary edema, especially with acute heart failure. The physical exam should also focus on comorbidities such as chronic kidney disease, diabetes mellitus, obesity, hypertension, AF, and COPD.

Laboratory studies include chemistry panels to determine the presence of renal, hepatic, or thyroid disease, glucose to 


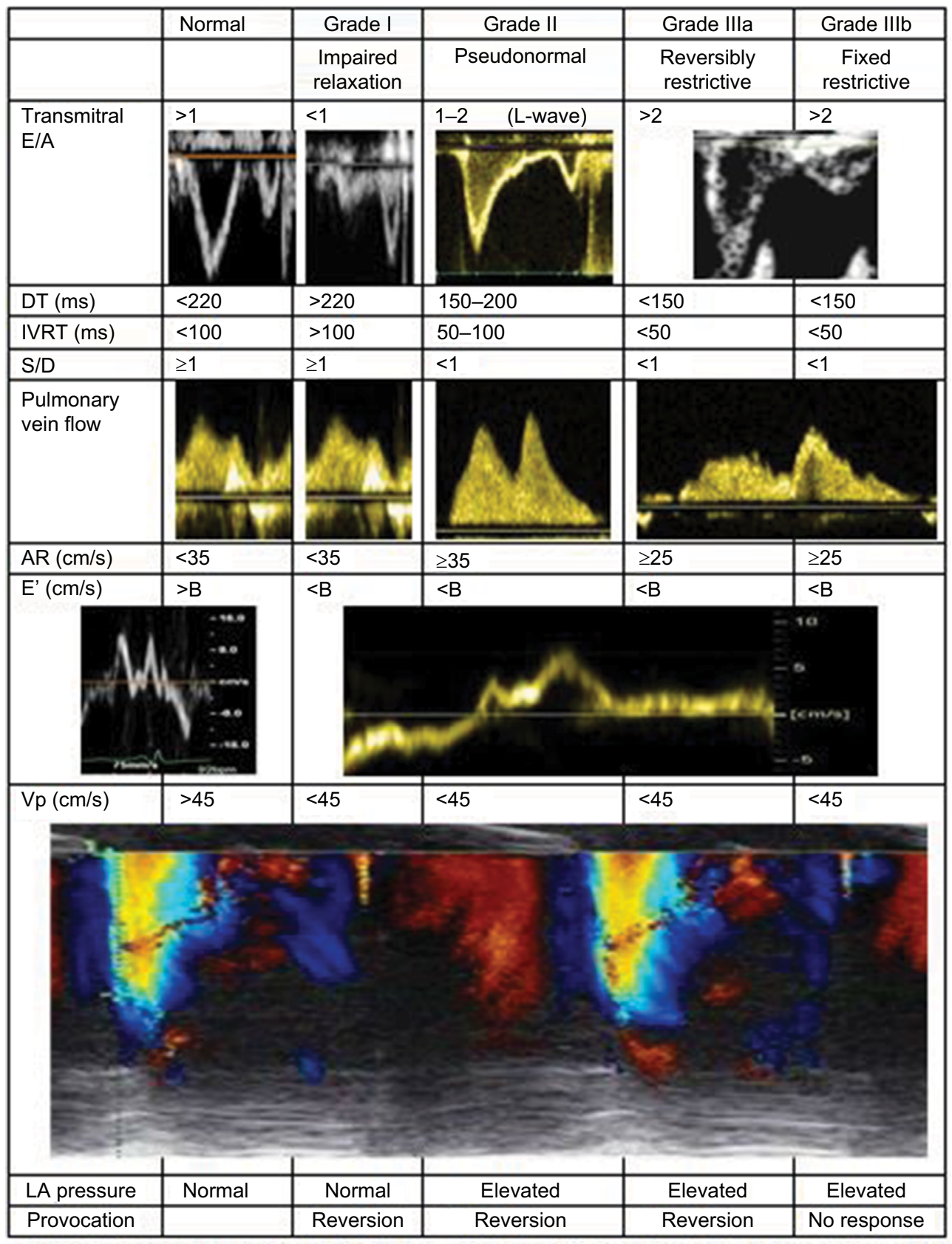

Figure 3 Echocardiographic Doppler findings.

Note: Data from Bishu et al. ${ }^{81}$

Abbreviations: A, peak transmitral Doppler late filling; AR, pulmonary venous atrial reversal velocity; $D$, diastolic pulmonary vein peak velocity; $D T$, deceleration time; E, peak transmitral Doppler early filling; E', tissue Doppler peak lateral mitral annular early diastolic velocity; IVRT, isovolumetric relaxation time; LA, left atrial; S, systolic pulmonary vein peak velocity; $V_{p}$, transmitral color M-mode propagation velocity.

detect diabetes, a complete blood count and ferritin to assess anemia, estimated glomerular filtration rate, and biomarkers.

The most well-studied biomarkers are the NP, BNP, and its inactive precursor, NT-proBNP. NT-proBNP remains in the circulation longer than BNP because it is not metabolized by neprilysin, the enzyme responsible for metabolizing BNP. These peptides are released in response to long-standing LV stretch or increased wall tension. Although patients with HFPEF have lower levels of BNP than the patients with
HFREF, for a given BNP level, the associated risk of allcause mortality and HF hospitalization is at least as high in patients with HFPEF. van Veldhuisen et al studied the use of BNP in patients with heart failure at baseline and over 18 months and related the BNP levels to a primary outcome, either a composite of all-cause mortality and HF hospitalization or to mortality alone. Even though the BNP levels were significantly higher in patients with HFREF, BNP was found to be a strong predictor of outcome in patients with HFPEF. 
BNP can also be used to differentiate HFPEF from comorbid conditions, such as obesity, anemia, and COPD, that can mimic the signs and symptoms of heart failure. In these conditions, the BNP may be normal or slightly elevated. ${ }^{115}$ BNP and NT-proBNP can be used to predict outcome and the response to treatment. A BNP of $>200 \mathrm{pg} / \mathrm{mL}$ or an NTproBNP $>300 \mathrm{pg} / \mathrm{mL}$ is suggestive of HFPEF.

Recently, other biomarkers resulting from neurohormonal activation and remodeling have been studied to aid in the diagnosis of HFPEF and as a guide to therapy. These include the biomarkers angiotensin II, renin, and aldosterone, to identify patients who are more likely to respond to RAAS inhibition, biomarkers of interstitial fibrosis including Galactin-3 (Gal-3) and soluble ST2 (sST-2), proinflammatory cytokines, and high-sensitivity troponins.

Gal-3 is a glycoprotein secreted by the activated macrophages that can induce myofibroblast proliferation, fibrogenesis, and tissue repair. ${ }^{116}$ Elevated plasma Gal-3 levels in patients with HFPEF were associated with adverse outcomes, independent of NP values. They have been demonstrated to have prognostic value, but were not superior to BNP, sST2, or C-reactive protein (CRP) as a predictor of mortality. ${ }^{117-119}$ The combination of BNP and Gal-3 was superior in risk prediction compared with either of the biomarkers alone, and Gal-3 was a more accurate predictor of poor outcomes in patients with HFPEF than in those with HFREF. ${ }^{120}$

$\mathrm{sST} 2$, a member of the IL-1 family, protects the myocardium against the adverse effects of overload and has been correlated with cardiac fibrosis. ${ }^{121}$ Studies regarding sST2 in patients with HFPEF have reported conflicting results. In a study of 195 consecutive patients, of which 76 had HFPEF, hospitalized with acute heart failure, sST2 alone or in combination with other biomarkers did not predict 6-month endpoints. ${ }^{122}$ But in another study of 447 patients, of which 197 had HFPEF, with 1-year follow-up, sST2 was an independent predictor of mortality, regardless of the LVEF. ${ }^{123}$ Wang et al demonstrated that sST2 was more accurate than NP in identifying the severity of DD. ${ }^{124}$ Gal-3 and sST2 may have the potential to identify subgroups of patients that may respond to antifibrotic therapies such as the mineralocorticoid receptor antagonists.

HFPEF is associated with a proinflammatory state producing endothelial and myocardial inflammation that is related to oxidative stress. Endothelial inflammation has a larger impact in HFPEF compared to HFREF ${ }^{28}$ Elevated levels of proinflammatory cytokines affect immune activation and coagulation, causing skeletal muscle wasting, reducing myocardial contractility, and inducing myocardial hypertrophy. ${ }^{125}$ The inflammatory cytokines, tumor necrosis factor alpha (TNF- $\alpha$ ), IL-6, CRP, and pentraxine, are markers for risk stratification in patients with HFPEF. Increases in TNF- $\alpha$ and its receptors, TNFR1 and TNFR2, are associated with advanced age, renal dysfunction, HTN, DM, peripheral vascular disease, and AF. ${ }^{126}$ Other biomarkers include MMP and TIMP, which have been found to be indicators of DD. ${ }^{127}$ Inflammatory biomarkers may be a measure of the risk for developing HFPEF in patients with $\mathrm{DD}^{128}$ and may be useful in assessing treatments targeting glycemic status, weight control, and exercise. The use of pleiotropic drugs, such as metformin and statins, may help improve the proinflammatory state in HFPEF. Metformin may be useful in treating prediabetic patients to prevent LV remodeling and cardiac fibrosis in HFPEF. ${ }^{129}$ Statins have anti-inflammatory, antihypertrophic, antifibrotic, and antioxidant effects and have been associated with reduced proinflammatory biomarker levels and improved echocardiographic measures of diastolic function and both cardiac and all-cause mortality. ${ }^{130}$

Coronary artery and coronary microvascular disease (CMVD) are the hallmarks of HFPEF. Advanced age, obesity, HTN, DM, and myocardial inflammation causing endothelial dysfunction are associated with CMVD, and the severity of myocardial fibrosis and LVH is correlated with the degree of CMVD. ${ }^{32}$ The biomarkers associated with CMVD include the high-sensitivity troponins. Elevated concentrations of these troponins are detectable even in the absence of unstable CAD and are associated with increased mortality. ${ }^{131}$ In a large multicenter registry, HFPEF patients had lower troponin levels than HFREF patients, suggesting less ongoing ischemic myocardial damage. ${ }^{132}$ Elevated high-sensitivity troponin has been found in the majority of HFPEF patients. It has been found to be associated with advanced age, DM, higher NT-proBNP levels, lower glomerular filtration rate (GFR), larger LA size, and larger LV volume and mass ${ }^{133}$ and may be used to differentiate the patients who would progress from LVH to HFPEF. ${ }^{134}$

Besides laboratory studies, the workup includes electrocardiography (ECG) and transthoracic echocardiography and in patients presenting with acute heart failure symptoms, chest radiography. Spirometry may be considered to rule out pulmonary disease as the cause of dyspnea. In patients with nondiagnostic studies, further testing with CMR, right or left heart catheterization, or provocation tests may be required.

ECG may reveal LVH and LA enlargement, as well as arrhythmias, ischemia, and conduction abnormalities. Abnormalities that may suggest DD include measures of delayed repolarization, including a prolonged $\mathrm{QT}_{\mathrm{c}}$ interval, a prolonged $\mathrm{T}$ wave peak to $\mathrm{T}$ wave end $(\mathrm{Tp}-\mathrm{Te})$ interval, 
$\mathrm{P}$ wave dispersion in the resting ECG, and a hump sign (upward deflection of the ST segment) during exercise ECG. A normal ECG does not exclude the presence of HFPEF. ${ }^{135,136}$

According to the American College of Cardiology Foundation/American Heart Association Task Force guidelines, transthoracic echocardiography is the test of choice for diagnosing DD and HFPEF. ${ }^{137}$ The hallmark of HFPEF is increased LA size and volume and Doppler echocardiographic measurements of impaired LV relaxation. These include the transmitral $\mathrm{E}$ and A velocity, the tissue Doppler $\mathrm{E}^{\prime}$, and the $\mathrm{E} / \mathrm{E}^{\prime}$ ratio. The $\mathrm{E}$ is the peak velocity during early diastole measured with pulsed wave Doppler, and $\mathrm{E}^{\prime}$ is the peak velocity of the septal or lateral mitral valve annulus. The transmitral E wave reflects the maximal gradient between the LA and left ventricle. It is dependent on ventricular compliance, but is affected by intravascular volume and LA pressure, both of which are increased in HFPEF. $E^{\prime}$ reflects the elastic recoil and active relaxation phase during diastole and correlates with $\tau$, the isovolumetric relaxation constant. The E/E' more closely reflects the LVEDP, which is affected by impaired relaxation, reduced compliance, and increased circulating volume. An $\mathrm{E} / \mathrm{E}^{\prime}>15$ is considered diagnostic for impaired diastolic function in symptomatic patients. ${ }^{111,138,139}$ In patients with an $\mathrm{E} / \mathrm{E}^{\prime}$ between 8 and 15 , HFPEF is present when the NT-proBNP is $>220 \mathrm{pg} / \mathrm{mL}$ or the BNP is $>200 \mathrm{pg} / \mathrm{mL}$.

For patients with inadequate echocardiographic windows, CMR may be useful as a surrogate. CMR is becoming the imaging method of choice for assessing LV systolic function. It can image in any plane, is more reproducible than 2D echocardiography, and allows for improved visualization of the myocardium. The use of CMR has been expanded to assess DD and to determine the etiology of nonischemic cardiomyopathy. LA volume is an important determinant of LV filling pressure and is used in the diagnosis, prognosis, and response to therapy in both DD and HFPEF. CMR, using volumetric short-axis Simpson's methods, is considered the gold standard for calculating LA volume. ${ }^{140}$ Through tissue characterization and late gadolinium enhancement, CMR is useful in differentiating between hypertrophic cardiomyopathy, hemochromatosis, sarcoidosis, and the presence, type, and prognosis of amyloid cardiomyopathy. CMR is also the gold standard for quantitating LV mass, which is associated with adverse cardiovascular outcomes and sudden death. CMR has been used to assess diastolic function by measuring the peak LV filling rates in early and late diastole, as well as the time to peak early LV filling. ${ }^{141}$ Newer applications of CMR in the assessment of DD include $T_{1}$ mapping to quantify diffuse myocardial fibrosis and to measure the extracellular volume fraction. The extracellular volume fraction correlates with the collagen volume fraction, the degree of fibrosis, and with $\beta$, the load-independent LV myocardial stiffness constant, and can be used to predict intrinsic LV stiffness in patients with DD or HFPEF. ${ }^{142}$

Blood flow velocity can be measured by velocity encoding or phase contrast $\mathrm{CMR},{ }^{143}$ allowing measurements of mitral valve inflow and pulmonary vein velocities. ${ }^{144}$ Strain, strain rate, and torsion can also be measured. CMR is also useful in determining the presence of pericardial disease.

When the clinical scenario and Doppler echocardiography are not sufficient to establish the diagnosis of HFPEF, or additional information is required to guide management, the patient may undergo invasive hemodynamic measurements of LV filling pressures. The gold standard is the direct measurement of LV pressure during left heart catheterization, which allows the direct measurement of the end-diastolic pressure and the calculation of $\tau$, the LV diastolic time constant, and $b$, the constant of chamber stiffness, measured as the slope of the pressure-volume curve. DD is suspected when $\tau$ is $>45 \mathrm{~ms}, b$ is $>0.27$, and the LV end-diastolic pressure is $>16$ mmHg. ${ }^{145}$ Right heart catheterization has been used instead of or in addition to left heart catheterization, when pulmonary hypertension in suspected. In the absence of mitral stenosis or pulmonary vein disease, the PCWP correlates with LV diastolic pressure and an elevated pulmonary artery mean pressure $>12-15 \mathrm{mmHg}$ is indicative of elevated $\mathrm{LV}$ filling pressures.

One of the challenges of assessing diastolic function is that the patient with HFPEF is often asymptomatic at rest and symptomatic with exertion or increased venous return. A normal LVEDP or normal E/E' may not exclude the diagnosis of HFPEF, as the degree of DD may be variable over time. Provocative tests are indicated when clinical suspicion is high, and include exercise or dobutamine Doppler echocardiography to measure diastolic reserve and intravenous fluid infusion or exercise during cardiac catheterization. ${ }^{69,146}$ In the absence of HFPEF, diastolic function should not change significantly with $\beta$-receptor stimulation. Pulmonary hypertension may also be unmasked.

Once HFPEF has been diagnosed, cardiopulmonary testing (CPX) can determine the functional limitations and other exercise abnormalities and may be used for prognosis. Information obtained from the CPX includes peak $\mathrm{VO}_{2}$, the $\mathrm{VE} / \mathrm{VCO}_{2}$ slope, heart rate and blood pressure changes, as well as subjective symptoms. The CPX can also detect concomitant issues such as ischemia, hypertensive 
response to exercise, chronotropic incompetence, COPD, and deconditioning.

\section{Therapeutic approach to HFPEF}

Therapy in heart failure is aimed at the amelioration of symptoms, improvement in function and quality of life, and prolonging survival. Unlike patients with HFREF, where treatment modalities have been found to prolong survival, treatment for patients with HFPEF has only been shown to improve symptoms and quality of life, but not survival. This may be because HFPEF is a more heterogeneous disorder than HFREF. Once the diagnosis of HFPEF has been established, the focus of treatment is aimed at the relief of symptoms, improved quality of life, and decreased hospitalization. This includes treating the comorbidities and concomitant factors that contributed to the development of HFPEF. Treatment includes managing hypertension, preferentially with inhibitors of the RAAS system such as the angiotensin converting enzyme inhibitors and the angiotensin receptor blockers, diabetes with metformin, morbid obesity, OSA, CAD, and hyperlipidemia. Small retrospective clinical studies have suggested that statins improve mortality outcomes, possibly due to their pleiotropic and anti-inflammatory properties. A recent meta-analysis has revealed a trend in decreased mortality in patients with HFPEF. Randomized clinical trials are still needed to determine the benefit of statins in reducing mortality in these patients. ${ }^{147,148}$

Symptomatic relief requires consuming a low-sodium diet and the use of loop diuretics to maintain a euvolemic state. These patients have impaired diastolic function and are sensitive to volume status. Hypervolemia can precipitate pulmonary edema and hypovolemia can reduce cardiac output due to the steep preload-cardiac output relationship at low volumes. A subgroup analysis of the CardioMEMS Heart Sensor Allows Monitoring of Pressure to Improve Outcomes in NYHA Class III Heart Failure Patients (CHAMPION) trial revealed that patients on diuretic therapy had a $50 \%$ reduction in the incidence of hospitalization for heart failure. ${ }^{149}$ Spironolactone has been demonstrated to have a small effect on E/E', LV mass, NT-proBNP levels, and reduced hospitalization for HF. ${ }^{150}$ To increase to duration of diastole and filling, b- or nondihydropyridine calcium channel blockers are a first-line therapy. Besides negative chronotropic effects, they have negative lusinotropic effects, which may be contributory to the increased prevalence of chronotropic incompetence in patients with HFPEF. 5 Along with pharmacologic therapy, promoting regular exercise and a healthy lifestyle is also important, especially in managing comorbidities. Exercise training in HFPEF improves exercise capacity and quality of life, but not mortality. ${ }^{151-153}$

Recently, other well-known medications studied in the treatment of HFPEF include the angiotensin receptor blockers candesartan and irbesartan, the phosphodiesterase-5 inhibitor sildenafil, and the slow sodium channel inhibitor ranolazine. Candesartan and irbesartan have been studied with disappointing results. Candesartan slightly reduced the hospitalization rates without affecting mortality, ${ }^{154}$ while irbesartan failed to improve cardiovascular outcomes or quality of life. ${ }^{155,156}$ Sildenafil, a phosphodiesterase-5 inhibitor, was thought to treat diastolic heart failure by increasing the supply of NO and reducing pulmonary hypertension, but showed conflicting results in two studies. The initial study by Guazzi et al, composed of elderly male patients with HFPEF and pulmonary hypertension, revealed that sildenafil significantly improved right atrial pressure, pulmonary artery pressure, right ventricular function, and quality of life. ${ }^{157}$ However, in the more recent Phosphodiesterase-5 Inhibition to Improve Clinical Status and Exercise Capacity in Diastolic Heart Failure (RELAX) study, with patients more representative of HFPEF, sildenafil failed to improve either aerobic exercise capacity or echocardiographic measures of DD. ${ }^{158}$ In the Ranolazine for the Treatment of Diastolic Heart Failure in Patients with Preserved Ejection Fraction (RALI-DHF) study, ranolazine, an antianginal agent that inhibits the slow sodium channel, was studied and found to have no significant effects on cardiac relaxation, filling pressure, or exercise tolerance. ${ }^{159}$

Promising agents currently being investigated include the combination angiotensin-perilysin inhibitor valsartan/ sacubitril, the $I_{f}$ channel blocker ivabradine, and the IL-1 blocker anakinra.

NPs are responsible for vasodilation and diuresis by suppressing sympathetic and RAAS activity in response to the pressure and volume overload seen in heart failure. They act by inhibiting the reabsorption of sodium in proximal and distal nephrons and by directly producing both arterial and venodilation. Their actions are terminated by neprilysin, an endopeptidase enzyme. Besides the NPs, neprilysin also metabolizes the vasodilators substance $\mathrm{P}$ and bradykinin, as well as the vasoconstrictors endothelin- 1 and angiotensin II. ${ }^{160}$ The net effect of neprilysin will depend on the balance between its actions of the vasodilating and vasoconstricting peptides. ${ }^{161}$

As heart failure progresses, there is a hyporesponsiveness and resistance to NPs due to downregulation of natriuretic receptors, altered signal transduction, and increased neprilysin activity. ${ }^{160}$ 
Valsartan is an angiotensin receptor blocker with an affinity for $\mathrm{AT}_{1}$ and blocks the actions of angiotensin II. Neprilysin inhibitors increase endogenous NP levels. Valsartan is added to sacubitril because the neprilysin inhibitors also inhibit the inactivation of angiotensin II. Valsartan/sacubitril decreased blood pressure, preferentially reducing systolic blood pressure, more than valsartan alone without an increase in angioedema. ${ }^{162,163}$ The Prospective Comparison of ARNI with ARB on Management of HFPEF (PARAMOUNT) trial, a Phase II trial comparing valsartan/sacubitril with valsartan in patients with symptomatic HFPEF and elevated NT-pro-BNP levels, found that with long-term therapy, valsartan/sacubitril reduced NT-pro-BNP levels, NYHA heart failure classification, blood pressure, and LA size, while preserving the renal function as seen by a decrease in creatinine and an increase in estimated glomerular filtration rate without a significant increase in angioedema. ${ }^{164}$ When switching patients from an ACE-I to valsartan/sacubitril, it is important to have at least a 36-hour ACE-I-free period before starting valsartan/sacubitril to prevent bradykinin accumulation and angioedema; but even with this drug-free interval, some patients may still develop angioedema.

LA size and LV mass were found to be independently associated with an increased risk of morbidity and mortality. ${ }^{35}$ By reducing the LA size and the risk of AF, valsartan/ sacubitril has a potential in treating patients with HFPEF.

A substudy analysis of the I-Preserve trial revealed an inverse association between heart rate and the incidence of hospitalization and mortality. ${ }^{165}$ Ivabradine, an $\mathrm{I}_{\mathrm{f}}$ channel blocker, acts on the sinus node to reduce the heart rate without affecting inotropy. A small Phase II study demonstrated that ivabradine significantly improved aerobic exercise capacity, as measured by peak $\mathrm{VO}_{2}$, ventilatory efficiency, as measured by the $\mathrm{VE} / \mathrm{VCO}_{2}$ ratio, myocardial relaxation, measured by $\mathrm{E}^{\prime}$, and diastolic cardiac reserve independent of the maximal heart rate response to exercise. ${ }^{166}$ In a small pilot crossover trial, anakinra, an IL-1 blocker, significantly reduced CRP levels and improved peak $\mathrm{VO}_{2}$ and $\mathrm{VE} / \mathrm{VCO}_{2}$. A Phase II study in patients with HFPEF treated with anakinra or placebo is ongoing.

Along with pharmacologic therapy, device therapy is currently being studied for patients with HFPEF. This includes rate-adaptive pacing to reduce chronotropic incompetence and exercise-induced dyspnea ${ }^{167}$ and LA pacing through a coronary sinus lead in patients with atrial dyssynchrony syndrome, characterized by interatrial conduction delay and increased LA stiffness. A pilot study demonstrated that LA pacing increased exercise tolerance, as measured by the 6-minute walk test distance, as well as improvements in LA and LV filling. ${ }^{168}$

Structural devices in development for the treatment of HFPEF include percutaneous interatrial shunts created to reduce LA pressure and exertional dyspnea and devices to mechanically assist LV relaxation. The InterAtrial Shunt Device (DC Devices, Inc., Tewksbury, MA, USA) is a percutaneously implanted $8 \mathrm{~mm}$ shunt tested in a pilot study of eleven patients with HFPEF and was found to reduce PCWP by $5.5 \mathrm{mmHg}$ at 30 days as well as NYHA symptoms in most patients. This device is currently being studied in the Reduce Elevated Left Atrial Pressure in Patients with Heart Failure (REDUCE LAP-HF) trial. ${ }^{169}$

The CORolla device (Corassist Cardiovascular Ltd, Herzliya, Israel) is composed of an elastic spring implanted in the LV apex that absorbs energy during ventricular systole and then applies expansion forces to the septum and lateral wall during diastole, increasing the diastolic suction and LV filling. Currently, this device requires surgical implantation, but a transapical approach is under development. ${ }^{170}$

\section{Future directions}

Future directions in the management of patients with HFPEF include aggressively treating comorbidities, developing new biomarkers to accurately determine the main pathologic process leading to $\mathrm{HF}$, and targeted strategies, instead of a "one-size-fits-all" regimen. Poorly treated comorbidities can cause death in these patients and aggressive treatment may delay the development of HFPEF.

Treatment strategies will be targeted to the dominant pathologic process, including passive stiffness, fibrosis, fluid retention, heart rate and rhythm, and pulmonary hypertension. Pacing and new devices, such as the CORolla, are also in development to treat the chronotropic incompetence and impaired relaxation associated with HFPEF.

\section{Conclusion}

HFPEF is a heterogeneous disorder characterized by multiple etiologies and diverse clinical presentations, complicated by a lack of universally accepted diagnostic criteria, resulting in a failure to improve morbidity and mortality, as compared to patients with HFREF. With ongoing research into the mechanisms of this disorder, new therapies are being developed to improve both symptoms and survival.

\section{Disclosure}

The authors report no conflicts of interest in this work. 


\section{References}

1. Mozaffarian D, Benjamin E, Go A, et al. Heart disease and stroke statistics - 2015 update. Circulation. 2014;131(4):e29-e322.

2. Aurigemma G, Zile MR, Gaasch WH. Contractile behavior of the left ventricle in diastolic heart failure: with emphasis on regional systolic function. Circulation. 2006;113(2):296-304.

3. Zile M, Brutsaert DL. New concepts in diastolic dysfunction and diastolic heart failure: part I: diagnosis, prognosis, and measurements of diastolic function. Circulation. 2002;105(11):1387-1393.

4. Hunt SA. ACC/AHA 2005 guideline update for the diagnosis management of chronic heart failure in the adult. ACC Curr J Rev. 2005;14(11):41-45

5. Abbate A, Arena R, Abouzaki N, et al. Heart failure with preserved ejection fraction: refocusing on diastole. Int J Cardiol. 2015;179: 430-440.

6. Kuznetsova T, Herbots L, Lopez B, et al. Prevalence of left ventricular diastolic dysfunction in a general population. Circ Heart Fail. 2009;2(2):105-112.

7. Kane G, Karon B, Mahoney D, et al. Progression of left ventricular diastolic dysfunction and risk of heart failure. JAMA. 2011; 306(8):856-863.

8. Owan T, Hodge D, Herges R, Jacobsen S, Roger V, Redfield M. Trends in prevalence and outcome of heart failure with preserved ejection fraction. N Engl J Med. 2006;355(3):251-259.

9. Sharp A, Tapp R, Francis D, et al. Ethnicity and left ventricular diastolic function in hypertension. J Am Coll Cardiol. 2008;52(12): 1015-1021.

10. Andersson C, Vasan RS. Epidemiology of heart failure with preserved ejection fraction. Heart Fail Clin. 2014;10(3):377-388.

11. Gelzinis T. New insights into diastolic dysfunction and heart failure with preserved ejection fraction. Semin Cardiothorac Vasc Anesth. 2013;18(2):208-217.

12. Satpathy C, Mishra T, Satpathy R, Satpathy H, Barone E. Diagnosis and management of diastolic dysfunction and heart failure. Am Fam Physician. 2006;73(5):841-846.

13. Newton J. Ethnicity and variation in prognosis for patients newly hospitalised for heart failure: a matched historical cohort study. Heart. 2005;91(12):1545-1550.

14. Neef SMaier L. Remodeling of excitation-contraction coupling in the heart: Inhibition of sarcoplasmic reticulum $\mathrm{Ca} 2+$ leak as a novel therapeutic approach. Curr Heart Fail Rep. 2007;4(1):11-17.

15. Mattiazzi A, Kranias E. CaMKII regulation of phospholamban and SR Ca2+ load. Heart Rhythm. 2011;8(5):784-787.

16. Anderson M. Oxidant stress promotes disease by activating CaMKII. J Mol Cell Cardiol. 2015;89:160-167.

17. van Heerebeek L, Franssen C, Hamdani N, Verheugt F, Somsen G, Paulus W. Molecular and cellular basis for diastolic dysfunction. Curr Heart Fail Rep. 2012;9(4):293-302.

18. Krüger M, Linke WA. Titin-based mechanical signalling in normal and failing myocardium. J Mol Cell. Cardiol. 2009;46(4):490-498.

19. Borbely A, Falcao-Pires I, van Heerebeek L, et al. Hypophosphorylation of the stiff N2B titin isoform raises cardiomyocyte resting tension in failing human myocardium. Circ Res. 2009;104(6):780-786.

20. Roe ND, Ren J. Oxidative activation of $\mathrm{Ca} 2+/$ calmodulin-activated kinase II mediates ER stress-induced cardiac dysfunction and apoptosis. Am J Physiol Heart Circ Physiol. 2013;304(6):H828-H839.

21. Borbely A. Cardiomyocyte stiffness in diastolic heart failure. Circulation. 2005;111(6):774-781.

22. Paulus W, Bronzwaer JG. Nitric oxide's role in the heart: control of beating or breathing? Am J Physiol Heart Circ Physiol. 2004;287(1):H8-H13.

23. Hammond J, Balligand J. Nitric oxide synthase and cyclic GMP signaling in cardiac myocytes: from contractility to remodeling. $\mathrm{J} \mathrm{Mol} \mathrm{Cell}$ Cardiol. 2012;52(2):330-340.

24. Kim H, Januzzi J. Natriuretic peptide testing in heart failure. Circulation. 2011;123(18):2015-2019.
25. Ritchie R, Irvine J, Rosenkranz A, et al. Exploiting cGMP-based therapies for the prevention of left ventricular hypertrophy: no and beyond. Pharmacol Ther. 2009;124(3):279-300.

26. Zile M, Baicu C, Gaasch W. Diastolic heart failure - abnormalities in active relaxation and passive stiffness of the left ventricle. $N$ Engl $J$ Med. 2004;350(19):1953-1959.

27. van Heerebeek L, Borbély A, Niessen HW, et al. Myocardial structure and function differ in systolic and diastolic heart failure. Circulation. 2006;113(16):1966-1973.

28. Paulus WJ, Tschöpe C. A novel paradigm for heart failure with preserved ejection fraction. J Am Coll Cardiol. 2013;62(4):263-271.

29. Kato S, Spinale F, Tanaka R, Johnson W, Cooper G, Zile M. Inhibition of collagen cross-linking: effects on fibrillar collagen and ventricular diastolic function. Am J Physiol. 1995;269(3):H863-868.

30. Rudolph A, Abdel-Aty H, Bohl S, oyé P, Zagrosek A, Dietz R, SchulzMenger J. Noninvasive detection of fibrosis applying contrast-enhanced cardiac magnetic resonance in different forms of left ventricular hypertrophy. J Am Coll Cardiol. 2009;53(3):284-291.

31. Kapila R, Mahajan R. Diastolic dysfunction. Contin Educ Anaesth Crit Care Pain. 2009;9(1):29-33.

32. Mohammed S, Borlaug B, Roger V, et al. Comorbidity and ventricular and vascular structure and function in heart failure with preserved ejection fraction: a community-based study. Circ Heart Fail. 2012;5(6):710-719.

33. Persson H, Lonn E, Edner M, et al. Diastolic dysfunction in heart failure with preserved systolic function: need for objective evidence: results from the CHARM echocardiographic substudy-CHARMES. J Am Coll Cardiol. 2007;49(6):687-694.

34. Abel E, Litwin S, Sweeney G. Cardiac remodeling in obesity. Physiol Rev. 2008;88(2):389-419.

35. Zile M, Gottdiener J, Hetzel S, et al. Prevalence and significance of alterations in cardiac structure and function in patients with heart failure and a preserved ejection fraction. Circulation. 2011;124(23): 2491-2501.

36. Katz D, Beussink L, Sauer A, Freed B, Burke M, Shah S. Prevalence, clinical characteristics, and outcomes associated with eccentric versus concentric left ventricular hypertrophy in heart failure with preserved ejection fraction. Am J Cardiol. 2013;112(8):1158-1164.

37. Massie BM, Carson PE, McMurray JJ, et al. Irbesartan in patients with heart failure and preserved ejection fraction. NEJOM. 2008;359: 2456-2467.

38. Shah A, Shah S, Anand I, et al. Cardiac structure and function in heart failure with preserved ejection fraction: baseline findings from the echocardiographic study of the treatment of preserved cardiac function heart failure with an aldosterone antagonist trial. Circ Heart Fail. 2013;7(1):104-115.

39. Kalogeropoulos A, Georgiopoulou V, Psaty B, et al. Inflammatory markers and incident heart failure risk in older adults. $\mathrm{J} \mathrm{Am} \mathrm{Coll}$ Cardiol. 2010;55(19):2129-2137.

40. Platis A, Yu Q, Moore D, Khojeini E, Tsau P, Larson D. The effect of daily administration of IL-18 on cardiac structure and function. Perfusion. 2008;23(4):237-242.

41. Díez J. Mechanisms of cardiac fibrosis in hypertension. J Clin Hypertens. 2007;9(7):546-550.

42. Asp M, Martindale J, Heinis F, Wang W, Metzger J. Calcium mishandling in diastolic dysfunction: Mechanisms and potential therapies. Biochim Biophys Acta. 2013;1833(4):895-900.

43. Kuschel M, Karczewski P, Hemple P, Schlegel W, Krause E, Bartel S. Ser16 prevails over Thr17 phospholamban phosphorylation in the beta-adrenergic regulation of cardiac relaxation. Am J Physiol. 1999;276(5):H1625-H1633.

44. Falcao-Pires I, Leite-Moreira AF. Diabetic cardiomyopathy: understanding the molecular and cellular basis to progress in diagnosis and treatment. Heart Fail Rev. 2011;17(3):325-344.

45. Witteles R, Fowler M. Insulin resistant cardiomyopathy. J Am Coll Cardiol. 2008;51(2):93-102.

46. Sengupta P, Khandheria B, Narula J. Twist and untwist mechanics of the left ventricle. Heart Fail Clin. 2008;4(3):315-324. 
47. Berk B, Fujiwara K, Lehoux S. ECM remodeling in hypertensive heart disease. J Clin Invest. 2007;117(3):568-575.

48. Berenji K, Drazner MH, Rothermel BA, Hill JA. Does load-induced ventricular hypertrophy progress to systolic heart failure? Am J Physiol Heart Circ Physiol. 2005;289(1):H8-H16.

49. Dostal D, Baker K. Angiotensin II stimulation of left ventricular hypertrophy in adult rat heart: mediation by the AT 1 receptor. $\mathrm{Am} J$ Hypertens. 1992;92(5):276-280.

50. Tschope C, Bock CT, Kasner M, et al. High prevalence of cardiac parvovirus B19 infection in patients with isolated left ventricular diastolic dysfunction. Circulation. 2005;111(7):879-886.

51. Belardinelli L, Shryock JC, Fraser H. Inhibition of the late sodium current as a potential cardioprotective principle: effects of the late sodium current inhibitor ranolazine. Heart. 2006;92(suppl_4):iv6-iv14.

52. Sossalla S, Fluschnik N, Schotola H, et al. Inhibition of elevated $\mathrm{Ca} 2+/$ calmodulin-dependent protein kinase II improves contractility in human failing myocardium. Circ Res. 2010;107(9):1150-1161.

53. Alagiakrishnan K, Banach M, Jones L, Datta S, Ahmed A, Aronow W. Update on diastolic heart failure or heart failure with preserved ejection fraction in the older adults. Ann Med. 2012;45(1):37-50.

54. Shenouda S, Widlansky M, Chen K, et al. Altered mitochondrial dynamics contributes to endothelial dysfunction in diabetes mellitus. Circulation. 2011;124(4):444-453.

55. Hart C, Meyer D, Tazelaar H, et al. Load versus humoral activation in the genesis of early hypertensive heart disease. Circulation. 2001;104(2):215-220.

56. Lam C, Roger V, Rodeheffer R, Borlaug B, Enders F, Redfield M. Pulmonary hypertension in heart failure with preserved ejection fraction. J Am Coll Cardiol. 2009;53(13):1119-1126.

57. Takimoto E, Belardi D, Tocchetti C, et al. Compartmentalization of cardiac beta-adrenergic inotropy modulation by phosphodiesterase type 5. Circulation. 2007;115(16):2159-2167.

58. van Heerebeek L, Hamdani N, Falcao-Pires I, et al. Low myocardial protein kinase $\mathrm{G}$ activity in heart failure with preserved ejection fraction. Circulation. 2012;126(7):830-839.

59. Westermann D, Kasner M, Steendijk P, et al. Role of left ventricular stiffness in heart failure with normal ejection fraction. Circulation. 2008;117(16):2051-2060.

60. Kawaguchi M, Hay I, Fetics B, Kass DA. Combined ventricular systolic and arterial stiffening in patients with heart failure and preserved ejection fraction: implications for systolic and diastolic reserve limitations. Circulation. 2003;107(5):714-720.

61. Ennezat PV, Lefetz Y, Maréchaux S, et al. Left ventricular abnormal response during dynamic exercise in patients with heart failure and preserved left ventricular ejection fraction at rest. $J$ Card Fail. 2008;14(6):475-480.

62. Kitzman D, Higginbotham M, Cobb F, Sheikh K, Sullivan M. Exercise intolerance in patients with heart failure and preserved left ventricular systolic function: Failure of the Frank-Starling mechanism. J Am Coll Cardiol. 1991;17(5):1065-1072.

63. Ha J, Choi D, Park S, et al. Left ventricular diastolic functional reserve during exercise in patients with impaired myocardial relaxation at rest. Heart. 2008;95(5):399-404.

64. Phan T, Shivu G, Abozguia K, Sanderson J, Frenneaux M. The pathophysiology of heart failure with preserved ejection fraction: from molecular mechanisms to exercise haemodynamics. Int $J$ Cardiol. 2012;158(3):337-343.

65. Chen C, Nakayama M, Nevo E, Fetics B, Maughan W, Kass D. Coupled systolic-ventricular and vascular stiffening with age. J Am Coll Cardiol. 1998;32(5):1221-1227.

66. Borlaug B, Melenovsky V, Russell S, et al. Impaired chronotropic and vasodilator reserves limit exercise capacity in patients with heart failure and a preserved ejection fraction. Circulation. 2006;114(20):2138-2147.

67. Maurer M, Burkhoff D, Fried L, Gottdiener J, King D, Kitzman D. Ventricular structure and function in hypertensive participants with heart failure and a normal ejection fraction. $\mathrm{J} \mathrm{Am} \mathrm{Coll} \mathrm{Cardiol.}$ 2007;49(9):972-981.
68. Ross J. Afterload mismatch and preload reserve: A conceptual framework for the analysis of ventricular function. Prog Cardiovasc Dis. 1976;18(4):255-264.

69. Borlaug B, Nishimura R, Sorajja P, Lam C, Redfield M. Exercise hemodynamics enhance diagnosis of early heart failure with preserved ejection fraction. Circ Heart Failure. 2010;3(5):588-595.

70. Chattopadhyay S, Alamgir M, Nikitin N, Rigby A, Clark A, Cleland J. Lack of diastolic reserve in patients with heart failure and normal ejection fraction. Circ Heart Fail. 2009;3(1):35-43.

71. Borlaug BA. The pathophysiology of heart failure with preserved ejection fraction. Nat Rev Cardiol. 2014;11(9):507-515.

72. Tilley DG, Rockman HA. Role of beta -adrenergic receptor signaling and desensitization in heart failure: new concepts and prospects for treatment. Expert Rev Cardiovascr Ther. 2006;4(3):417-432.

73. Naga Prasad SV, Perrino C, Rockman HA. Role of phosphoinositide 3-kinase in cardiac function and heart failure. Trends Cardiovasc Med. 2003;13(5):206-212.

74. Zile M, Kjellstrom B, Bennett T, et al. Effects of exercise on left ventricular systolic and diastolic properties in patients with heart failure and a preserved ejection fraction versus heart failure and a reduced ejection fraction. Circ Heart Fail. 2013;6(3):508-516.

75. Haykowsky M, Brubaker P, John J, Stewart K, Morgan T, Kitzman D. Determinants of exercise intolerance in elderly heart failure patients with preserved ejection fraction. $J \mathrm{Am}$ Coll Cardiol. 2011;58(3):265-274.

76. Guazzi M, Myers J, Arena R. Cardiopulmonary exercise testing in the clinical and prognostic assessment of diastolic heart failure. $\mathrm{JAm} \mathrm{Coll}$ Cardiol. 2005;46(10):1883-1890.

77. Guazzi M, Cahalin L, Arena R. Cardiopulmonary exercise testing as a diagnostic tool for the detection of left-sided pulmonary hypertension in heart failure. J Cardiac Fail. 2013;19(7):461-467.

78. Shah S, Heitner J, Sweitzer N, et al. Baseline characteristics of patients in the treatment of preserved cardiac function heart failure with an aldosterone antagonist trial. Circ Heart Fail. 2012;6(2):184-192.

79. McMurray J, Carson P, Komajda M, et al. Heart failure with preserved ejection fraction: clinical characteristics of 4133 patients enrolled in the I-PRESERVE trial. Eur J Heart Fail. 2008;10(2):149-156.

80. McMurray J, Adamopoulos S, Anker S, et al. ESC guidelines for the diagnosis and treatment of acute and chronic heart failure 2012: The task force for the diagnosis and treatment of acute and chronic heart failure 2012 of the European Society of Cardiology. Developed in collaboration with the Heart Failure Association (HFA) of the ESC. Eur Heart J. 2012;33(14):1787-1847.

81. Bishu K, Deswal A, Chen H, et al. Biomarkers in acutely decompensated heart failure with preserved or reduced ejection fraction. $\mathrm{Am}$ Heart J. 2012;164(5):763-770.e3.

82. Filippatos G, Teerlink J, Farmakis D, et al. Serelaxin in acute heart failure patients with preserved left ventricular ejection fraction: results from the RELAX-AHF trial. Eur Heart J. 2013;35(16): 1041-1050.

83. Naito M, David D, Michelson E, Schaffenburg M, Dreifus L. The hemodynamic consequences of cardiac arrhythmias: evaluation of the relative roles of abnormal atrioventricular sequencing, irregularity of ventricular rhythm and atrial fibrillation in a canine model. Am Heart J. 1983;106(2):284-291.

84. Shantsila E, Shantsila A, Blann A, Lip G. Left ventricular fibrosis in atrial fibrillation. Am J Cardiol. 2013;111(7):996-1001.

85. Olsson L, Swedberg K, Ducharme A, et al. Atrial fibrillation and risk of clinical events in chronic heart failure with and without left ventricular systolic dysfunction. J Am Coll Cardiol. 2006;47(10):1997-2004.

86. Campbell RT, McMurray J. Comorbidities and differential diagnosis in heart failure with preserved ejection fraction. Heart Fail Clin. 2014;10(3):481-501.

87. MacDonald M, Petrie M, Varyani F, et al. Impact of diabetes on outcomes in patients with low and preserved ejection fraction heart failure: an analysis of the Candesartan in Heart failure: Assessment of Reduction in Mortality and morbidity (CHARM) programme. Eur Heart J. 2008;29(11):1377-1385. 
88. Somers V, White D, Amin R, et al. Sleep apnea and cardiovascular disease: an American Heart Association/American College of Cardiology Foundation scientific statement from the American Heart Association Council for high blood pressure Research Professional Education Committee, Council on Clinical Cardiology, Stroke Council, and Council on Cardiovascular Nursing In Collaboration with the National Heart, Lung, and Blood Institute National Center on sleep disorders research (National Institutes of Health). Circulation. 2008;118(10):1080-1111.

89. Pascual M, Vicente T, Hernández AM, Tébar FJ, Valdés M. Effects of isolated obesity on systolic and diastolic left ventricular function. Heart. 2003;89(10):1152-1156.

90. Brouwers F, de Boer R, van der Harst P, et al. Incidence and epidemiology of new onset heart failure with preserved vs. reduced ejection fraction in a community-based cohort: 11-year follow-up of PREVEND. Eur Heart J. 2013;34(19):1424-1431.

91. Ho J, Lyass A, Lee D, et al. Predictors of new-onset heart failure: differences in preserved versus reduced ejection fraction. Circ Heart Fail. 2012;6(2):279-286.

92. Lam C, Lyass A, Kraigher-Krainer E, et al. Cardiac dysfunction and noncardiac dysfunction as precursors of heart failure with reduced and preserved ejection fraction in the community. Circulation. 2011;124(1):24-30

93. Ather S, Chan W, Bozkurt B, et al. Impact of noncardiac comorbidities on morbidity and mortality in a predominantly male population with heart failure and preserved versus reduced ejection fraction. $\mathrm{JAm}$ Coll Cardiol. 2012;59(11):998-1005.

94. Maisel A, Mueller C, Adams K, et al. State of the art: using natriuretic peptide levels in clinical practice. Eur J Heart Fail. 2008;10(9):824-839.

95. Penicka M, Vanderheyden M, Bartunek J. Diagnosis of heart failure with preserved ejection fraction: role of clinical Doppler echocardiography. Heart. 2013;100(1):68-76.

96. Selmeryd J, Henriksen E, Leppert J, Hedberg P. Interstudy heterogeneity of definitions of diastolic dysfunction severely affects reported prevalence. Eur Heart J Cardiovasc Imaging. Epub 2015 Sep 15.

97. Chapman C, Ewer S, Kelly A, Jacobson K, Leal M, Rahko P. Classification of left ventricular diastolic function using American Society of echocardiography guidelines: agreement among echocardiographers. Echocardiography. 2013:30(9):1022-1031.

98. Unzek S, Popovic Z, Marwick T; Diastolic Guidelines Concordance Investigators. Effect of recommendations on interobserver consistency of diastolic function evaluation. JACC Cardiovasc Imaging. 2011;4(5):460-467.

99. Kloch-Badelek M, Kuznetsova T, Sakiewicz W, et al. Prevalence of left ventricular diastolic dysfunction in European populations based on cross-validated diagnostic thresholds. Cardiovasc Ultrasound. 2012;10(1):10.

100. AlJaroudi W, Thomas J, Rodriguez L, Jaber W. Prognostic value of diastolic dysfunction. Cardiol Rev. 2014;22(2):79-90.

101. Lester S, Tajik A, Nishimura R, Oh J, Khandheria B, Seward J. Unlocking the mysteries of diastolic function. $\mathrm{J} \mathrm{Am} \mathrm{Coll} \mathrm{Cardiol.}$ 2008;51(7):679-689.

102. Tsang T, Barnes M, Gersh B, Bailey K, Seward J. Left atrial volume as a morphophysiologic expression of left ventricular diastolic dysfunction and relation to cardiovascular risk burden. Am J Cardiol. 2002;90(12):1284-1289.

103. Lam C, Han L, Ha J, Oh J, Ling L. The mitral L wave: A marker of pseudonormal filling and predictor of heart failure in patients with left ventricular hypertrophy. J Am Soc Echocardiogr. 2005;18(4) 336-341.

104. Oh J, Tajik J. The return of cardiac time intervals: the phoenix is rising. J Am Coll Cardiol. 2003;42(8):1471-1474.

105. Brun P, Tribouilloy C, Duval A, et al. Left ventricular flow propagation during early filling is related to wall relaxation: A color M-mode Doppler analysis. J Am Coll Cardiol. 1992;20(2):420-432.

106. Stugaard M, Brodahl U, Torp H, Ihlen H. Abnormalities in left ventricular filling in patients with coronary artery disease: assessment by colour M-mode Doppler technique. Eur Heart J. 1994;15(3):318-327.
107. Stugaard M, Smiseth O, Risøe C, Ihlen H. Intraventricular early diastolic velocity profile during acute myocardial ischemia: a color M-mode Doppler echocardiographic study. J Am Soc Echocardiogr. 1995;8(3):270-279.

108. Nagueh S, Middleton K, Kopelen H, Zoghbi W, Quiñones MA. Doppler tissue imaging: a noninvasive technique for evaluation of left ventricular relaxation and estimation of filling pressures. $\mathrm{J} \mathrm{Am} \mathrm{Coll}$ Cardiol. 1997;30(6):1527-1533.

109. Ommen S, Nishimura R, Appleton C, et al. Clinical utility of Doppler echocardiography and tissue Doppler imaging in the estimation of left ventricular filling pressures: a comparative simultaneous Dopplercatheterization study. Circulation. 2000;102(15):1788-1794.

110. Wang J, Khoury D, Thohan V, Torre-Amione G, Nagueh S. Global diastolic strain rate for the assessment of left ventricular relaxation and filling pressures. Circulation. 2007;115(11):1376-1383.

111. Firstenberg M, Levine B, Garcia M, et al. Relationship of echocardiographic indices to pulmonary capillary wedge pressures in healthy volunteers. J Am Coll Cardiol. 2000;36(5):1664-1669.

112. González-Vilchez F, Ayuela J, Ares M, Mata NS, González AG, Durán RM. Comparison of Doppler echocardiography, color M-mode Doppler, and Doppler tissue imaging for the estimation of pulmonary capillary wedge pressure. JAm Soc Echocardiogr. 2002;15(10):1245-1250.

113. Oh J, Park S, Nagueh S. Established and novel clinical applications of diastolic function assessment by echocardiography. Circ Cardiovasc Imaging. 2011;4(4):444-455.

114. Zuccal ̃̃ G, Marzetti E, Cesari M, et al. Correlates of cognitive impairment among patients with heart failure: results of a multicenter survey. Am J Med. 2005;118(5):496-502.

115. van Veldhuisen D, Linssen G, Jaarsma T, et al. B-type natriuretic peptide and prognosis in heart failure patients with preserved and reduced ejection fraction. J Am Coll Cardiol. 2013;61(14):1498-1506.

116. Yang R, Rabinovich G, Liu F. Galectins: structure, function and therapeutic potential. Expert Rev Mol Med. 2008;10:e17.

117. de Boer R, Lok D, Jaarsma T, et al. Predictive value of plasma galectin-3 levels in heart failure with reduced and preserved ejection fraction. Ann Med. 2010;43(1):60-68.

118. Edelmann F, Holzendorf V, Wachter R, et al. Galectin-3 in patients with heart failure with preserved ejection fraction: results from the Aldo-DHF trial. Eur J Heart Fail. 2014;17(2):214-223.

119. Srivatsan V, George M, Shanmugam E. Utility of galectin-3 as a prognostic biomarker in heart failure: where do we stand? Eur J Prev Cardiol. 2014;22(9):1096-1110.

120. van der Velde A, Gullestad L, Ueland T, et al. Prognostic value of changes in galectin-3 levels over time in patients with heart failure: data from CORONA and COACH. Circ Heart Fail. 2013;6(2):219-226.

121. Shah RV, Januzzi JL Jr. ST2: a novel remodeling biomarker in acute and chronic heart failure. Curr Heart Fail Rep. 2010;7(1):9-14.

122. Friões F, Lourenço P, Laszczynska O, et al. Prognostic value of sST2 added to BNP in acute heart failure with preserved or reduced ejection fraction. Clin Res Cardiol. 2015;104(6):491-499.

123. Manzano-Fernández S, Mueller T, Pascual-Figal D, Truong Q, Januzzi J. Usefulness of soluble concentrations of interleukin family member ST2 as predictor of mortality in patients with acutely decompensated heart failure relative to left ventricular ejection fraction. Am J Cardiol. 2011;107(2):259-267.

124. Wang Y, Yu C, Chiu F, Tsai CT, Lai LP, Hwang JJ, Lin JL. Soluble ST2 as a biomarker for detecting stable heart failure with a normal ejection fraction in hypertensive patients. J Cardiac Fail. 2013;19(3):163-168.

125. Paulus W. Cytokines and heart failure. Heart Fail Monit. 2000;1(2): 50-56.

126. Putko B, Wang Z, Lo J, et al. Circulating levels of tumor necrosis factoralpha receptor 2 are increased in heart failure with preserved ejection fraction relative to heart failure with reduced ejection fraction: evidence for a divergence in pathophysiology. PLoS One. 2014;9(6):e99495.

127. Collier P, Watson C, Voon V, et al. Can emerging biomarkers of myocardial remodelling identify asymptomatic hypertensive patients at risk for diastolic dysfunction and diastolic heart failure?. Eur J Heart Fail. 2011;13(10):1087-1095. 
128. Haass M, Kitzman D, Anand I, et al. Body mass index and adverse cardiovascular outcomes in heart failure patients with preserved ejection fraction: results from the Irbesartan in Heart Failure with Preserved Ejection Fraction (I-PRESERVE) trial. Circ Heart Fail. 2011;4(3):324-331.

129. Ladeiras-Lopes R, Fontes-Carvalho R, Bettencourt N, Sampaio F, Gama V, Leite-Moreira A. Novel therapeutic targets of metformin: metabolic syndrome and cardiovascular disease. Expert Opin Ther Targets. 2015;19(7):869-877.

130. D’Elia E, Vaduganathan M, Gori M, Gavazzi A, Butler J, Senni M. Role of biomarkers in cardiac structure phenotyping in heart failure with preserved ejection fraction: critical appraisal and practical use. Eur J Heart Fail. 2015;17(12):1231-1239.

131. de Lemos J, Drazner M, Omland T, et al. Association of troponin $\mathrm{T}$ detected with a highly sensitive assay and cardiac structure and mortality risk in the general population. JAMA. 2010;304(22):2503.

132. Greenberg B. Heart failure preserved ejection fraction with coronary artery disease. J Am Coll Cardiol. 2014;63(25):2828-2830.

133. Packer M, McMurray J, Desai A, et al. Angiotensin receptor neprilysin inhibition compared with enalapril on the risk of clinical progression in surviving patients with heart failure. Circulation. 2014;131(1):54-61.

134. Seliger SL, de Lemos J, Neeland IJ, et al. Older adults, "malignant" left ventricular hypertrophy, and associated cardiac-specific biomarker phenotypes to Identify the differential risk of new-onset reduced versus preserved ejection fraction heart failure: CHS. JACC Heart Fail. 2015;3(6):445-455.

135. Sauer A, Wilcox J, Andrei A, Passman R, Goldberger J, Shah S. Diastolic electromechanical coupling: association of the ECG T-peak to T-end interval with echocardiographic markers of diastolic dysfunction. Circ Arrhythm Electrophysiol. 2012;5(3):537-543.

136. Taha T, Sayed K, Saad M, Samir M. How accurate can electrocardiogram predict left ventricular diastolic dysfunction? Egypt Heart J. 2016;68(2):117-123.

137. Hunt S, Abraham W, Chin M, et al. 2009 Focused update incorporated into the ACC/AHA 2005 guidelines for the diagnosis and management of heart failure in adults: a report of the American College of Cardiology Foundation/American Heart Association Task Force on practice guidelines: developed in collaboration with the International Society for Heart and Lung Transplantation. Circulation. 2009;119(14):e391-e479.

138. Nagueh S. Echocardiographic assessment of left ventricular relaxation and cardiac filling pressures. Curr Heart Fail Rep. 2009;6(3):154-159.

139. Little WC, Oh JK. Echocardiographic evaluation of diastolic function can be used to guide clinical care. Circulation . 2009;120(9):802-809.

140. Vassiliou V, Patel H, Rosen S, et al. Left atrial dilation in patients with heart failure and preserved ejection fraction: Insights from cardiovascular magnetic resonance. Int J Cardiol. 2016;210:158-160.

141. Gonzalez JA, Kramer CM. Role of imaging techniques for diagnosis, prognosis and management of heart failure patients: cardiac magnetic resonance. Curr Heart Fail Rep. 2015;12(4):276-283.

142. Rommel K, von Roeder M, Latuscynski K, et al. Extracellular volume fraction for characterization of patients with heart failure and preserved ejection fraction. J Am Coll Cardiol. 2016;67(15):1815-1825.

143. Hartiala J, Mostbeck G, Foster E, et al. Velocity-encoded cine MRI in the evaluation of left ventricular diastolic function: measurement of mitral valve and pulmonary vein flow velocities and flow volume across the mitral valve. Am Heart J. 1993;125(4):1054-1066.

144. Flachskampf F, Biering-Sørensen T, Solomon S, Duvernoy O, Bjerner T, Smiseth O. Cardiac imaging to evaluate left ventricular diastolic function. JACC Cardiovasc Imaging. 2015;8(9):1071-1093.

145. Kindermann M. How to diagnose diastolic heart failure: a consensus statement on the diagnosis of heart failure with normal left ventricular ejection fraction by the heart failure and Echocardiography Associations of the European Society of Cardiology. Eur Heart J. 2007;28(21):2686-2686.

146. Burgess M, Jenkins C, Sharman J, Marwick T. Diastolic stress echocardiography: hemodynamic validation and clinical significance of estimation of ventricular filling pressure with exercise. $\mathrm{J} \mathrm{Am} \mathrm{Coll}$ Cardiol. 2006;47(9):1891-1900.
147. Gomez-Soto F, Romero S, Bernal J, et al. Mortality and morbidity of newly diagnosed heart failure with preserved systolic function treated with $\hat{\mathrm{I}}^{2}$-blockers: A propensity-adjusted case-control populational study. Int J Cardiol. 2011;146(1):51-55.

148. Tehrani F, Morrissey R, Phan A, Chien C, Schwarz E. Statin therapy in patients with diastolic heart failure. Clin Cardiol. 2009:33(4): E1-5.

149. Adamson P, Abraham W, Bourge R, et al. Wireless pulmonary artery pressure monitoring guides management to reduce decompensation in heart failure with preserved ejection fraction. Circ Heart Fail. 2014;7(6):935-944.

150. Pitt B, Pfeffer M, Assmann S, et al. Spironolactone for heart failure with preserved ejection fraction. N Engl J Med. 2014;370(15): 1383-1392.

151. Kitzman D, Brubaker P, Herrington D, et al. Effect of endurance exercise training on endothelial function and arterial stiffness in older patients with heart failure and preserved ejection fraction. $\mathrm{J} \mathrm{Am} \mathrm{Coll}$ Cardiol. 2013;62(7):584-592.

152. Nolte K, Herrmann-Lingen C, Wachter R, et al. Effects of exercise training on different quality of life dimensions in heart failure with preserved ejection fraction: the Ex-DHF-P trial. Eur J Prev Cardiol. 2014;22(5):582-593.

153. Edelmann F, Gelbrich G, Düngen HD, et al. Exercise training improves exercise capacity and diastolic function in patients with heart failure with preserved ejection fraction. J Am Coll Cardiol. 2011;58(17): 1780-1791.

154. Yusuf S, Pfeffer M, Swedberg K, et al. Effects of candesartan in patients with chronic heart failure and preserved left-ventricular ejection fraction: the CHARM-preserved trial. Lancet. 2003;362(9386):777-781.

155. Massie B, Carson P, McMurray J, et al. Irbesartan in patients with heart failure and preserved ejection fraction. $N$ Engl J Med. 2008;359(23):2456-2467.

156. Rector T, Carson P, Anand I, et al. Assessment of long-term effects of irbesartan on heart failure with preserved ejection fraction as measured by the Minnesota Living with Heart Failure Questionnaire in the irbesartan in heart failure with preserved systolic function (I-PRESERVE) trial. Circ Heart Fail. 2012;5(2):217-225.

157. Guazzi M, Vicenzi M, Arena R, Guazzi M. Pulmonary hypertension in heart failure with preserved ejection fraction: a target of phosphodiesterase-5 inhibition in a 1-year study. Circulation. 2011; 124(2):164-174.

158. Redfield M, Chen H, Borlaug B, et al. Effect of phosphodiesterase-5 inhibition on exercise capacity and clinical status in heart failure with preserved ejection fraction. JAMA. 2013;309(12):1268.

159. Jacobshagen C, Belardinelli L, Hasenfuss G, Maier L. Ranolazine for the treatment of heart failure with preserved ejection fraction: background, aims, and design of the RALI-DHF study. Clin Cardiol. 2011;34(7):426-432.

160. Andersen MB, Simonsen U, Wehland M, Pietsch J, Grimm D. LCZ696 (valsartan/sacubitril) - a possible new treatment for hypertension and heart failure. Basic Clin Pharmacol Toxicol. 2015;118(1): 14-22.

161. Langenickel T, Dole W. Angiotensin receptor-neprilysin inhibition with LCZ696: a novel approach for the treatment of heart failure. Drug Discov Today. 2012;9(4):e131-e139.

162. Kario K, Sun N, Chiang F, et al. Efficacy and safety of LCZ696, a first-in-class angiotensin receptor neprilysin inhibitor, in Asian patients with hypertension novelty and significance. Hypertension. 2014;63(4):698-705.

163. Ruilope L, Dukat A, Bohm M, Lacourcière Y, Gong J, Lefkowitz M. Blood-pressure reduction with LCZ696, a novel dual-acting inhibitor of the angiotensin II receptor and neprilysin: a randomised, double-blind, placebo-controlled, active comparator study. Lancet. 2010;375(9722):1255-1266.

164. Solomon S, Zile M, Pieske B, et al. The angiotensin receptor neprilysin inhibitor LCZ696 in heart failure with preserved ejection fraction: a phase 2 double-blind randomised controlled trial. Lancet. 2012;380(9851):1387-1395. 
165. Böhm M, Perez A, Jhund P, et al. Relationship between heart rate and mortality and morbidity in the irbesartan patients with heart failure and preserved systolic function trial (I-Preserve). Eur J Heart Fail. 2014;16(7):778-787.

166. Cotter G, Moshkovitz Y, Milovanov O, et al. Acute heart failure: a novel approach to its pathogenesis and treatment. Eur J Heart Fail. 2002;4(3):227-234

167. Kass D, Kitzman D, Alvarez G. The restoration of chronotropic competence in heart failure patients with normal ejection fraction (RESET) study: rationale and design. J Card Fail. 2010;16(1):17-24.
168. Laurent G, Eicher J, Mathe A, et al. Permanent left atrial pacing therapy may improve symptoms in heart failure patients with preserved ejection fraction and atrial dyssynchrony: a pilot study prior to a national clinical research programme. Eur J Heart Fail. 2013;15(1):85-93.

169. Gustafsson F, Malek F, Neuzil P, Sondergaard L, Walton T. TCT-77 midterm results of a novel interatrial shunt therapy for heart failure and preserved ejection fraction. J Am Coll Cardiol. 2014;64(11):B23.

170. Tannenbaum Sayer G. Advances in the pathophysiology and treatment of heart failure with preserved ejection fraction. Curr Opin Cardiol. 2015;30(3):250-258.
Research Reports in Clinical Cardiology

\section{Publish your work in this journal}

Research Reports in Clinical Cardiology is an international, peerreviewed, open access journal publishing original research, reports, editorials, reviews and commentaries on all areas of cardiology in the clinic and laboratory. The manuscript management system is completely online and includes a very quick and fair peer-review system.

\section{Dovepress}

Visit http://www.dovepress.com/testimonials.php to read real quotes from published authors. 